\title{
Les oratoires dans l'espace rural varois
} Joël Candau

\section{Citer ce document / Cite this document :}

Candau Joël. Les oratoires dans l'espace rural varois. In: Le Monde alpin et rhodanien : revue régionale d'ethnologie, $n^{\circ} 2-$ 3/1985. pp. 83-107;

http://www.persee.fr/doc/mar_0758-4431_1985_num_13_2_1265

Document généré le 20/11/2017 


\section{Les oratoires dans l'espace rural varois}

Dans l'ensemble des objets qui servent de supports aux recherches sur ce qu'il est d'usage d'appeler, par commodité, la religion populaire, les oratoires constituent une source peut-être trop négligée.

Certes, il existe de précieux inventaires qui facilitent les investigations de l'ethnographe sur le terrain, mais ceux-ci se limitent généralement - et c'est déjà beaucoup - à un recensement des monuments dans une région ou un département donnés, après un développement obligé sur la filiation, jamais réellement démontrée, de ces édicules avec les montjoies, les monuments païens, voire avec les monolithes préhistoriques. Ces ouvrages, inspirés par les théories de la survivance, ne voient le plus souvent dans les oratoires que «de touchants témoignages de la foi de nos aïeux" (1), s'interdisant du même coup toute possibilité d'appréhender le sens qu'ils peuvent avoir pour les croyants - ou les non croyants - d'aujourd'hui.

Dans le cadre d'une thèse de doctorat (2), nous nous sommes efforcé de mener une recherche approfondie sur ce type de monuments dans le département du Var. Différents sujets ont été abordés : le palmarès des intercesseurs (3), la distribution calendaire des fêtes des titulaires, leurs emblèmes et leurs pouvoirs, l'orientation des édicules, la motivation des fondateurs d'oratoires, l'attitude du clergé, etc. Dans cet article sera développé un aspect de ce travail qui, d'une certaine façon, a constitué le fil conducteur de la recherche : l'insertion spatiale des oratoires dans deux cantons varois, celui du Beausset et celui de Rians (4). En bref, il s'est agi,

(1) V. PIGUILLEM, "Les oratoires des Pyrénées orientales", Archistra, 1974, n 13, p. 8.

(2) Thèse pour le Doctorat de $3^{\text {e }}$ Cycle en Ethnologie, soutenue le 4 juillet 1984 à l'Université de Nice devant un jury présidé par M. Michel Vovelle et rassemblant MM. les Professeurs Paul Raybaut, Richard Pottier (rapporteur) et M. Raymond Eches, Maître-assistant à l'U.E.R. Lettres et Sciences humaines de Nice.

(3) La question des dédicaces méritant un article à elle seule, nous nous abstiendrons à dessein de l'évoquer ici. Livrons simplement une information brute sur le panthéon constitué par les titulaires des monuments. On connaît 767 dédicaces sur les 838 oratoires varois recensés. La Vierge arrive en tête avec 261 citations, suivie de saint Joseph (49), saint Jean-Baptiste (35), le Christ (27), saint Pierre (25), sainte Marie-Magdeleine (24), sainte Anne (23), saint Antoine de Padoue (18), saint Eloi (16), saint Michel et saint Louis (13), saint Roch (12), saint Marc (11), saint Etienne (10), sainte Thérèse de l'Enfant Jésus (9), saint François d'Assise et saint Pons (8), saint Clair et saint André (7), quatre saints cités six fois, quatre cités cinq fois, quatre autres cités quatre fois, seize saints mentionnés trois fois, treize saints pourvus de deux titulatures et cinquante-sept intercesseurs cités une seule fois.

(4) Les cantons du Beausset (16903 habitants en 1982, 23425 ha) et de Rians (6 119 habitants en 1982, 34673 ha) se trouvent respectivement aux confins sud-ouest et nord-ouest du département du Var. Le canton du Beausset s'insère pour une bonne part dans la bande littorale varoise. Celui de Rians, borné au nord par le Verdon, appartient au Haut Var. 
tout au long de l'enquête, de ne pas se satisfaire de la question : "Pourquoi des oratoires ?", mais de l'accompagner de l'interrogation : "Pourquoi là ?" Cette problématique, nous le verrons, introduit inévitablement l'idée d'un rapport possible entre ces édicules et la conjuration du malheur biologique et écologique.

\section{Définition}

Qu'est-ce qu'un oratoire ? Bien qu'il n'y ait pas deux monuments strictement identiques, on constate une variation sur un même thème, un même modèle, avec des nuances architecturales aussi bien entre régions qu'à l'intérieur d'un département.

L'oratoire, le plus souvent quadrangulaire, cylindrique quelquefois, a une base de $70 \mathrm{~cm}$ de côté en moyenne et une hauteur variant généralement entre 1,8 et 2.5 mètres (5). Le monument s'appuie parfois sur un socle haut de 10 à $20 \mathrm{~cm}$ dont les côtés débordent de quelques centimètres ceux du fût. La niche, abri du saint titulaire, fait pour cette raison la spécificité du monument (6). Elle est rectangulaire, cintrée ou ogivale, avec des dimensions moyennes de $60 \mathrm{~cm}$ de hauteur sur $40 \mathrm{~cm}$ de large et $40 \mathrm{~cm}$ de profondeur. L'édicule, ordinairement en pierres (moellons, pierres sèches ou taillées, jointoyées ou non), parfois en briques maçonnées, avec ou sans enduit, se termine par un toit sans charpente, en dôme, ou pyramidal, ou bien encore à une ou deux pentes, fait de tuiles ou de pierres plates, le tout souvent surmonté d'une croix, habituellement en fer et ouvragée. L'oratoire porte quelquefois une épigraphe : inscription de la date d'érection, dédicace, etc. La statue du saint, occasionnellement honorée de fleurs des champs ou de fleurs artificielles a, en règle générale, une hauteur de 20 à 30 centimètres.

Selon les régions de France, on désigne cette "oraison de pierre» (7), cette prière qui "monte du sol» (8) du nom de mariette (Eure-et-Loir), d'ouradour (Limousin), de rougadou ou de pilon (de pieloun, en Provence), de reposoir, de capelette (dans le Nord), de santète (Hautes-Pyrénées) ou, tout simplement, de chapelle. Toutefois, le terme oratoire est le plus souvent utilisé, ce qui s'avère bien gênant pour le chercheur engagé dans des investigations bibliographiques ou archivistiques. Cette appellation, en effet, n'a pas partout la même acception et s'applique à la miniature pieuse (9) de 10 à 30 centimètres comme à la chapelle dans laquelle on peut célébrer l'office ou, encore, à l'édicule étudié ici. Dès lors, toute quête documentaire s'avère hasardeuse : il devient malaisé d'évaluer la diffusion de l'oratoire dans le temps et dans l'espace à partir des seules sources écrites si l'on n'est jamais sûr de son identification à une époque ou dans un lieu donnés.

Dès 1939, Van Gennep mettait un peu d'ordre en distinguant :

(5) On peut trouver des monuments de plus grande taille, tels que l'oratoire Saint-Martin à Rians qui dépasse les quatre mètres.

(6) Doit-on ranger parmi les oratoires les niches murales ? Bien que les Amis des oratoires à Aixen-Provence répondent par la négative, le fichier de cette association les prend en compte. VAN GENNEP procède de même dans un article paru en 1939 : "Patronages, chapelles et oratoires de la HauteMaurienne. Etude statistique et critique», Revue d'Histoire de l'Eglise de France, t. XXV, 1939, pp. 145-182. Pour notre part, nous avons recensé les niches murales dont les dimensions sont au moins égales à la dimension moyenne des niches des monuments ordinaires.

(7) Pierre IRIGOIN, Les oratoires de Provence, Aix-en-Provence, Amis des oratoires, 1940, p. 6.

(8) Louis PORCHERON, "Les oratoires de Provence», Ciels et terres de Provence, Marseille, éd. "Quo vadis", 1938, p. 410.

(9) Ce type d'objet, visible au Musée des Arts et Traditions populaires, est en céramique ou en terre cuite. Il représente une niche abritant un saint, surmontée d'un crucifix. 


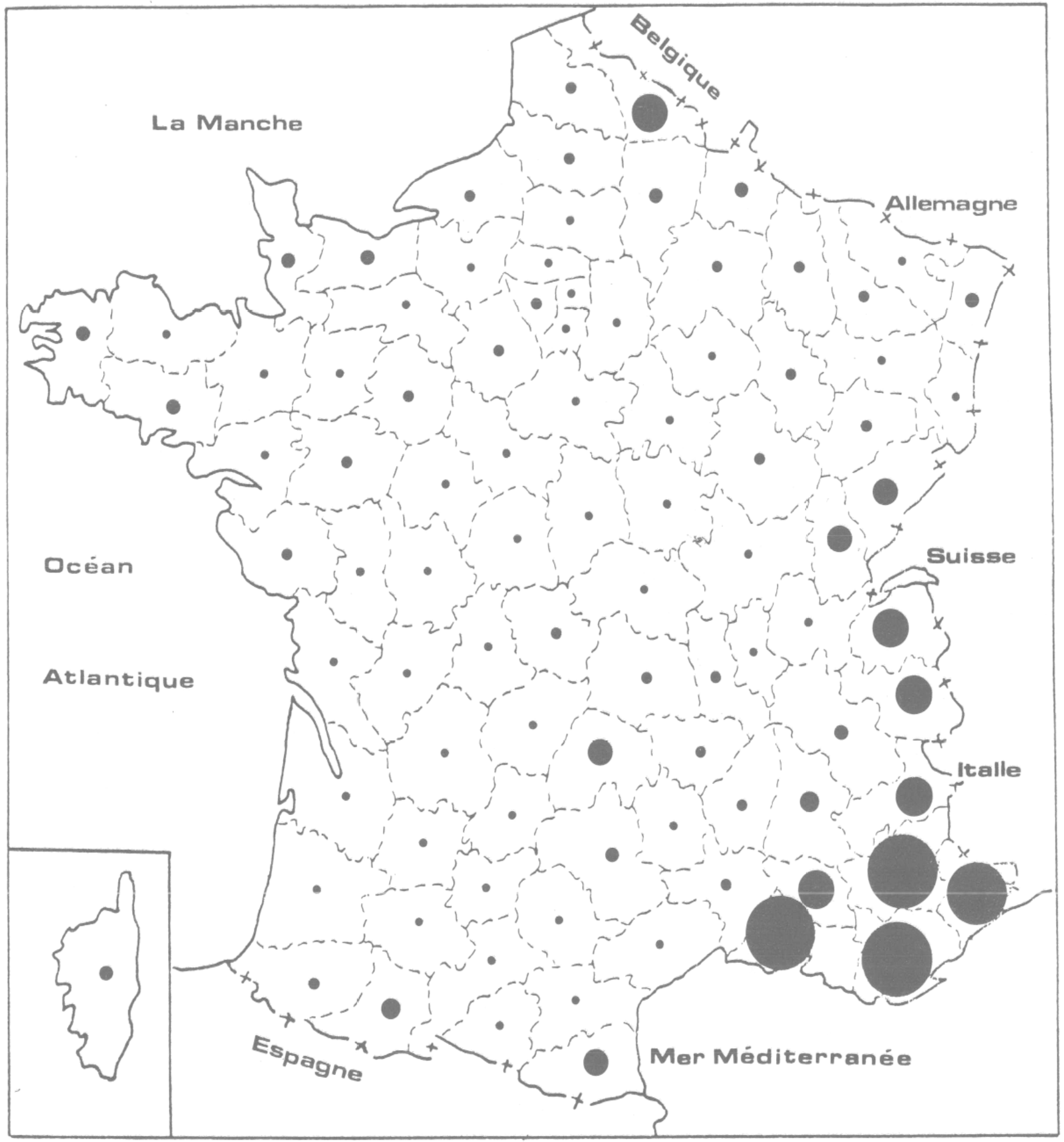

Carte $n^{\circ}$ 1. Densité des oratoires dans les départements français (données : octobre 1983).

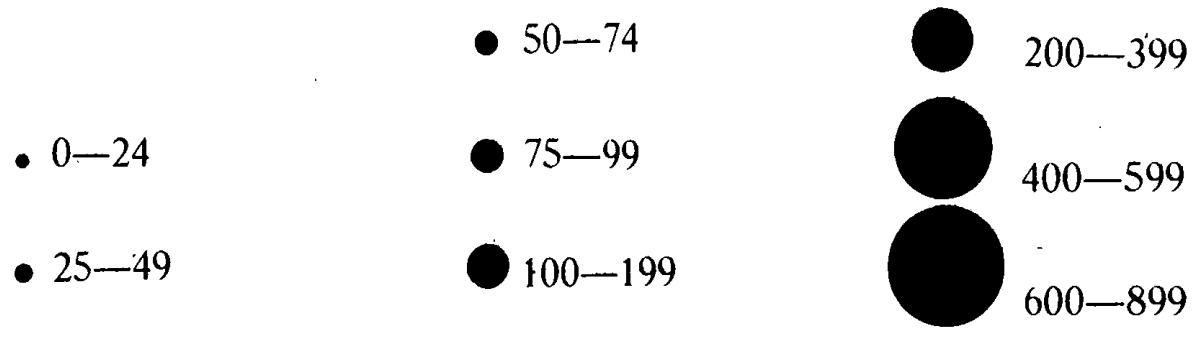




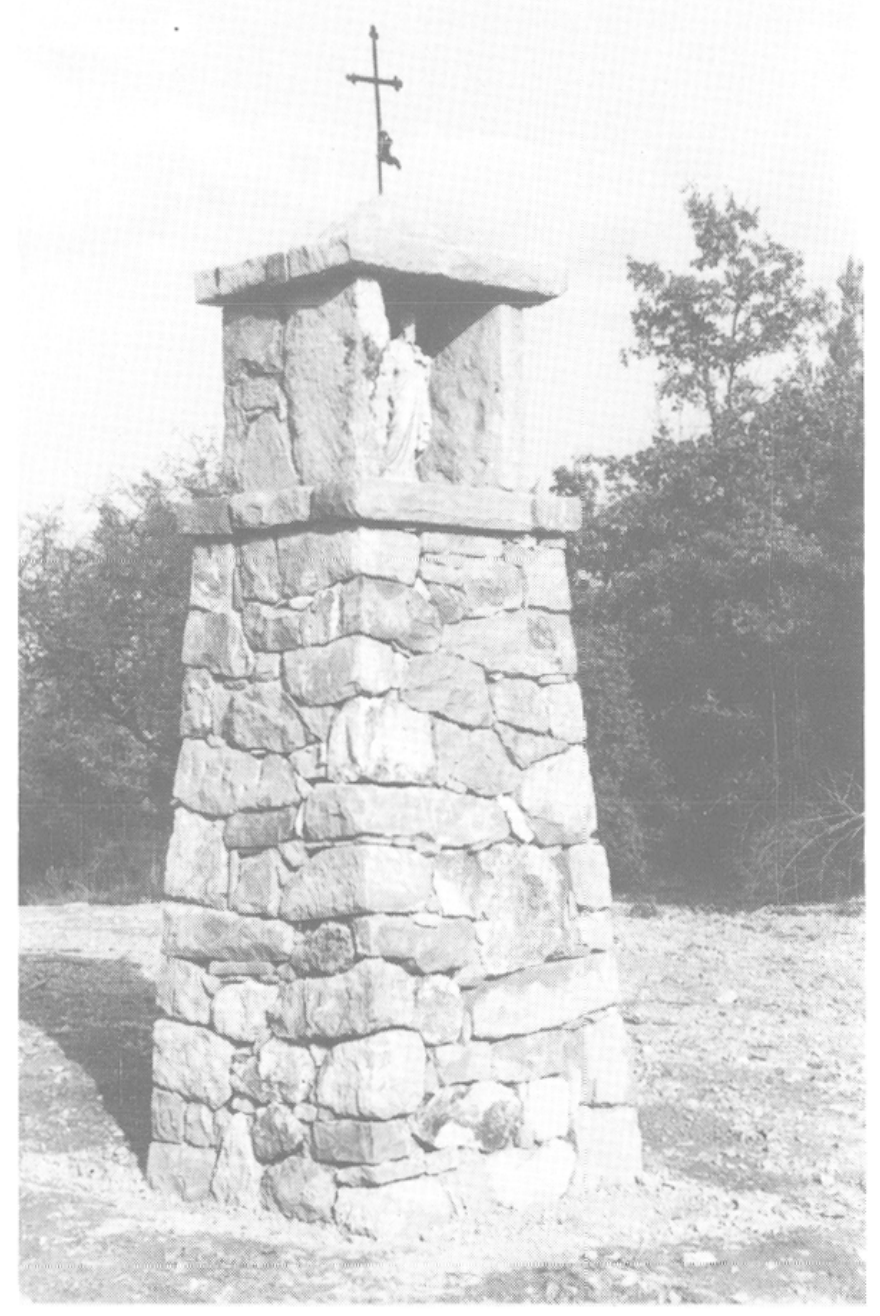

1. Saint-Marc, Rians. Oratoire ancien, restauré en 1984. Localisation : saltus, bord d'un chemin. Orientation : $180^{\circ}$. En pierres; niche sur corniche; dalles sur le toit surmontées d'une demi-sphère de pierre. Dimensions : $253 h \times 98 l \times 98 p(a ̀$ la base $)$ et $75 l \times 74 p(a u$ sommet du pilier). Niche $: 52 h \times 42 l \times 52 p$.

2. Notre-Dame, Saint-Cyr. Oratoire ancien (1907). Localisation : saltus. Orientation : $330^{\circ}$. En briques sur socle avec niche cintrée sur corniche moulurée renfermant une statuette protégée par une grille. L'intérieur de la niche est peint en bleu. Toit pyramidal sur corniche. surmonté d'une croix tréflée en fer. Statuette de Notre-Dame de Lourdes à côté d'un crucifix. Dimensions : $238 h \times 52 l \times 52 p$. Niche $: 63 h \times 38 l \times 46 p$.

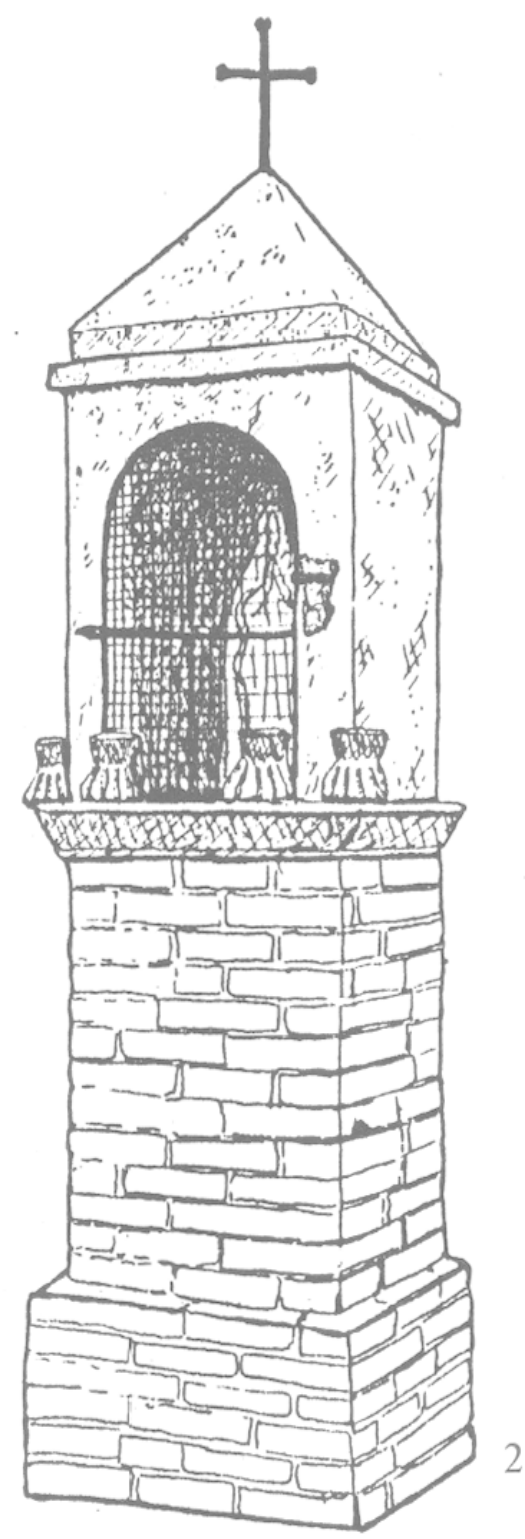




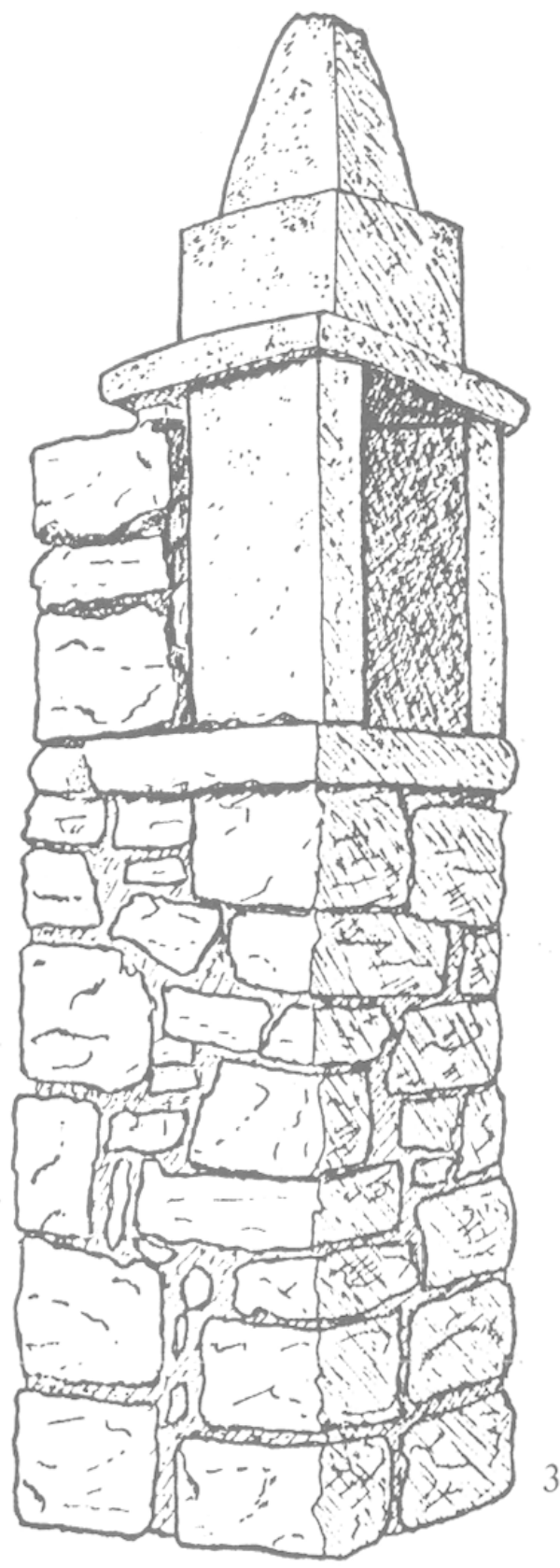

3. Sainte Cécile, Rians. Oratoire ancien (1768 ou 1769). Localisation: terrain situé dans l'ager jadis, en voie d'urbanisation aujourd hui. Orientation : $40^{\circ}$. Pilier en pierres : niche formée de quatre dalles en pierres de taille, vide: toit en tronc de pyramide sur socle carré. Lancienne croix de fer a disparu. Dimensions: $294 h \times 63 l \times 64 p$. Niche $: 54 h \times 37 l \times 29 p$.

4. Sainte-Thérèse de l'Enfant-Jésus, La Verdière. Oratoire ancien (1950). Localisation : ager, bord de route. Orientation : $190^{\circ}$. Pilier en pierres avec niche à trois ouvertures cintrées, surmontée d'un toit pyramidal terminé par une croix de fer. Ouvertures de la niche vitrées et grillagées. Plaque de marbre posée sur le devant du pilier avec l'inscription : "Année sainte 1950". Dimensions : $273 h \times 65 l \times 69 p$. Niche : $58 h \times$ $36 l \times 56 p$.

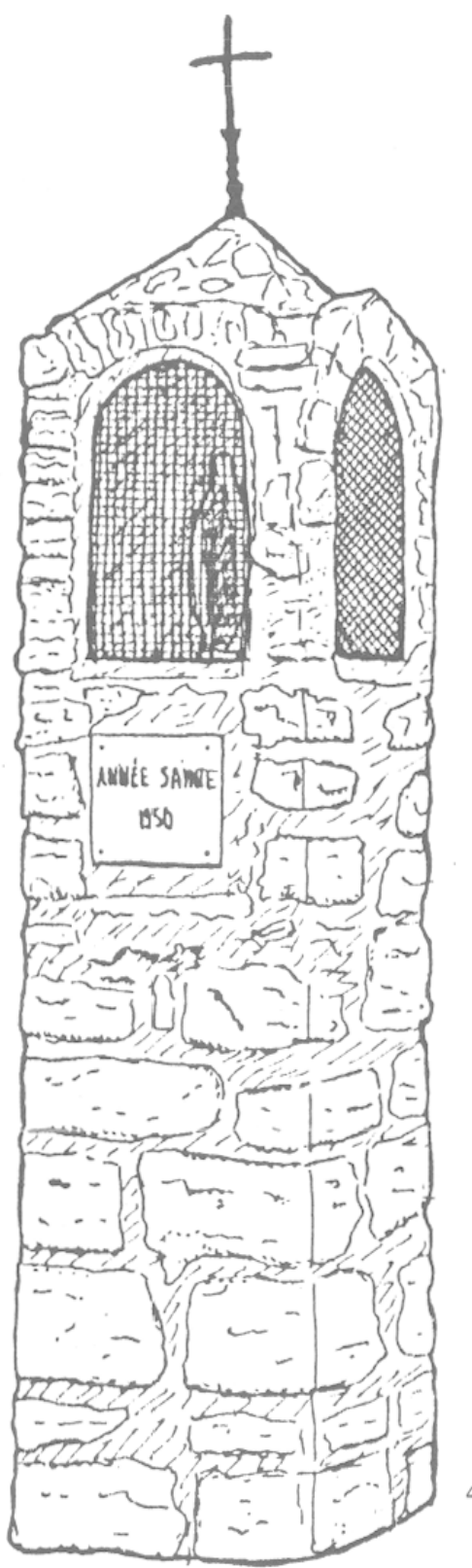


«Quatre catégories de sanctuaires qui portent le nom du saint : $1^{\circ}$ l'église ou la chapelle paroissiale; $2^{\circ}$ la chapelle dans l'église, avec autel personnel, ou joint à un autre, ou au maître-autel ; $3^{\circ}$ la chapelle rurale, consacrée, où le prêtre a le droit de dire la messe $; 4^{\circ}$ le simple oratoire devant lequel les fidèles prient sans qu'il y ait lieu à messe périodique ou occasionnelle». (10)

Seule l'enquête de terrain permet d'effectuer ces distinctions. L'Association des Amis des oratoires (11), à qui l'on doit la quasi totalité des différents inventaires régionaux, s'est attelée à cette tâche considérable depuis plus de cinquante ans. Elle a entrepris le recensement de tous les oratoires français, en s'aidant du principe classificatoire suivant :

"Dans une chapelle on entre ; dans un oratoire, on ne peut pas entrer». (12).

Pour abrupte qu'elle soit, la distinction nous paraît bonne. Par souci de clarté, et comme le souhaitent les $A$ mis des oratoires, on devrait employer le terme oratoire exclusivement pour désigner des monuments où il est impossible de pénétrer et de célébrer le culte. C'est en tout cas la convention que nous avons adoptée lors de notre enquête dans le Var.

\section{Premières localisations}

Etudier les oratoires revient à interroger une réalité régionale. Dans le sud-est de la France, un observateur attentif ne manquera pas d'être étonné par leur densité. Cette région, en effet, privilégie ce type de monuments. Huit départements (13) enferment 3668 oratoires, soit $56 \%$ des 6851 édicules recensés par les $A m i s$ des oratoires dans l'ensemble du pays (14). A lui seul, le département du Var en regroupe $838(15)$.

Comment expliquer un tel phénomène ? Cette question pose le "problème irritant» (16) des aires folkloriques et culturelles. Si nous sommes incapable d'y répondre, notons néanmoins que les disparités régionales, évidentes sur la carte $n^{\circ} 1$, devraient sans doute être nuancées si l'on prenait en compte l'existence d'autres monuments, tels que les croix rurales ou les oratoires-fontaines.

De même éprouvons-nous des difficultés à interpréter la carte de densité des oratoires varois (Carte $n^{\circ}$ 2). Le plus grand nombre de monuments à l'ouest du département bouscule notre connaissance d'une Provence occidentale traditionnelle-

(10) Arnold VAN GENNEP, ibid., p. 146.

(11) Les Amis des oratoires, Maison des Agriculteurs, 22 Avenue Henri-Pontier, 13626 Aix-en-Provence.

(12) René PAUPLIN, Les oratoires du Vaucluse, Aix-en-Provence, Les Amis des oratoires, 1970. p. 6.

(13) Alpes-Maritimes, Alpes-de-Haute-Provence, Bouches-du-Rhône, Hautes-Alpes, Savoie, Haute-Savoie, Var, Vaucluse.

(14) Données : fin octobre 1983.

(15) Recension de M. Louis JANVIER (Inventaire des oratoires $d u$ Var, Aix-en-Provence, Amis des oratoires, 1982, 121 p.), actualisée fin octobre 1983 à partir des archives de l'association des Amis des oratoires et corrigée en fonction de notre propre inventaire dans les cantons du Beausset et de Rians.

(16) Robert CHANAUD, «Folklore et religion dans le diocèse de Grenoble à la fin du XVIIe siècle : les visites pastorales de Monseigneur Le Camus", Le Monde Alpin et Rhodanien, n 1-4/1977, Religion populaire, p. 68 . 


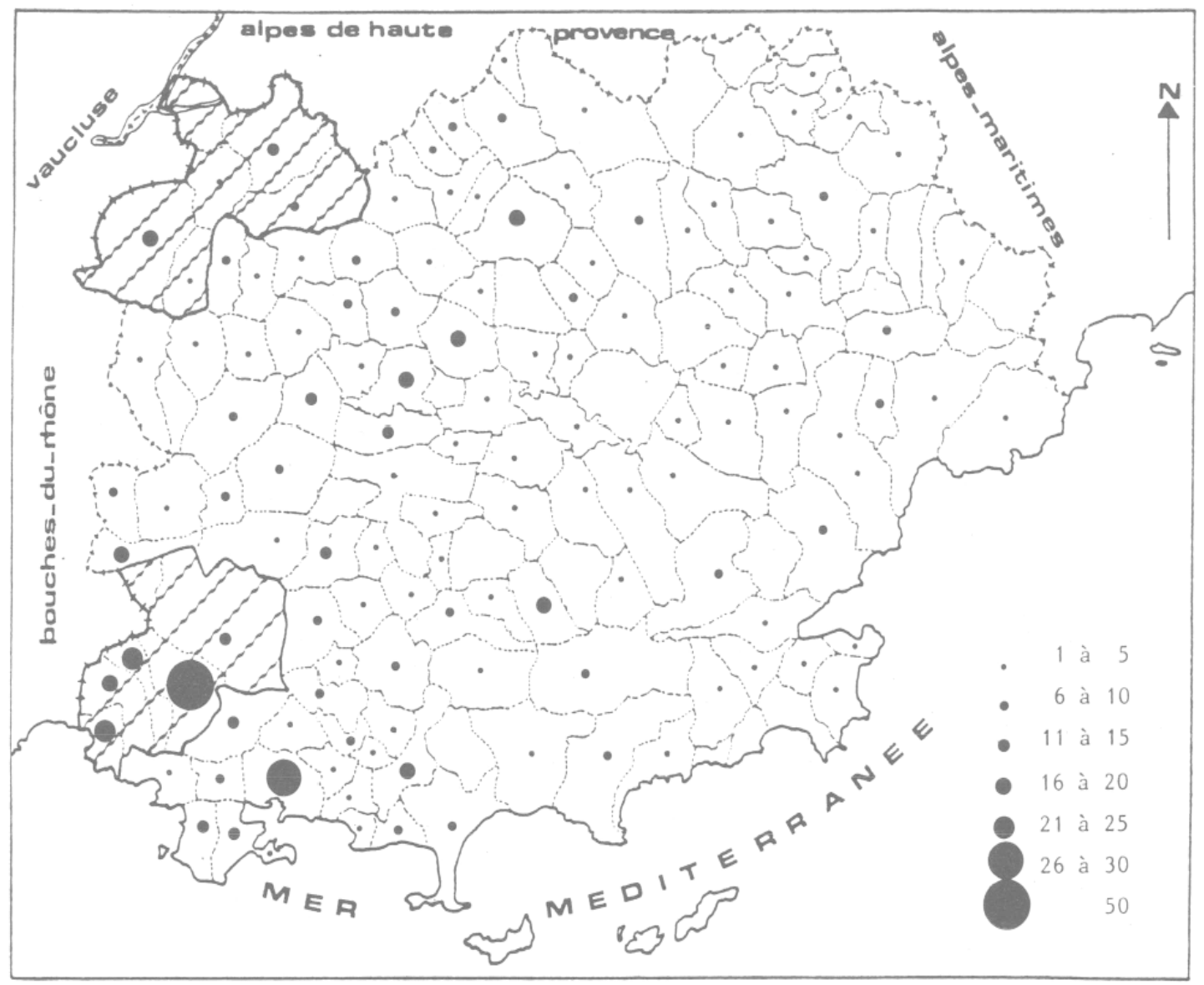

Carte $n^{\circ}$ 2. Densité des oratoires dans les communes du département du Var. Les cantons du Beausset et de Rians figurent en hachuré.

ment moins dévote que la région orientale. Mais souvenons-nous que le Var occidental abrite aussi la plus forte concentration d'ex-voto (17).

A l'intérieur de cette partie du département, le Var maritime apparaît privilégié ce qui, là encore, n'entre pas dans nos schémas habituels. Doit-on chercher une explication dans les écarts démographiques ? Dans ce cas, on comprend mal que le canton de Rians, relativement peu peuplé, ait plus d'oratoires que, par exemple, celui de Hyères (18).

Comment dépasser notre incompréhension actuelle de cette carte ? Nous aurions tendance à penser que d'autres travaux de géographie religieuse permettraient d'y voir plus clair : cartographie de tous les lieux de culte varois, de l'ensemble des itinéraires de pèlerinages, etc. Dans l'état actuel de nos recherches, nous sommes forcé d'en rester là.

Dans les deux cantons du Beausset et de Rians, les cartes d'implantation des édicules (Cartes $n^{\circ} 3$ et 4 ) livrent une première information, assez triviale : les oratoires sont situés dans les zones d'occupation humaine. La partie des deux cartes cantonales vide de monuments correspond essentiellement, pour Le Beausset, au

(17) Bernard COUSIN, Le Miracle et le quotidien. Les ex-voto provençaux, images d'une société, Aix-en-Provence, Thèse pour le doctorat d'Etat, Université de Provence, p. 187.

(18) Par ailleurs, nous n'avons trouvé aucune corrélation entre la densité des oratoires et le type de structures agraires (petites exploitations dans le Var maritime, moyennes ou grandes propriétés dans le Haut-Var). 


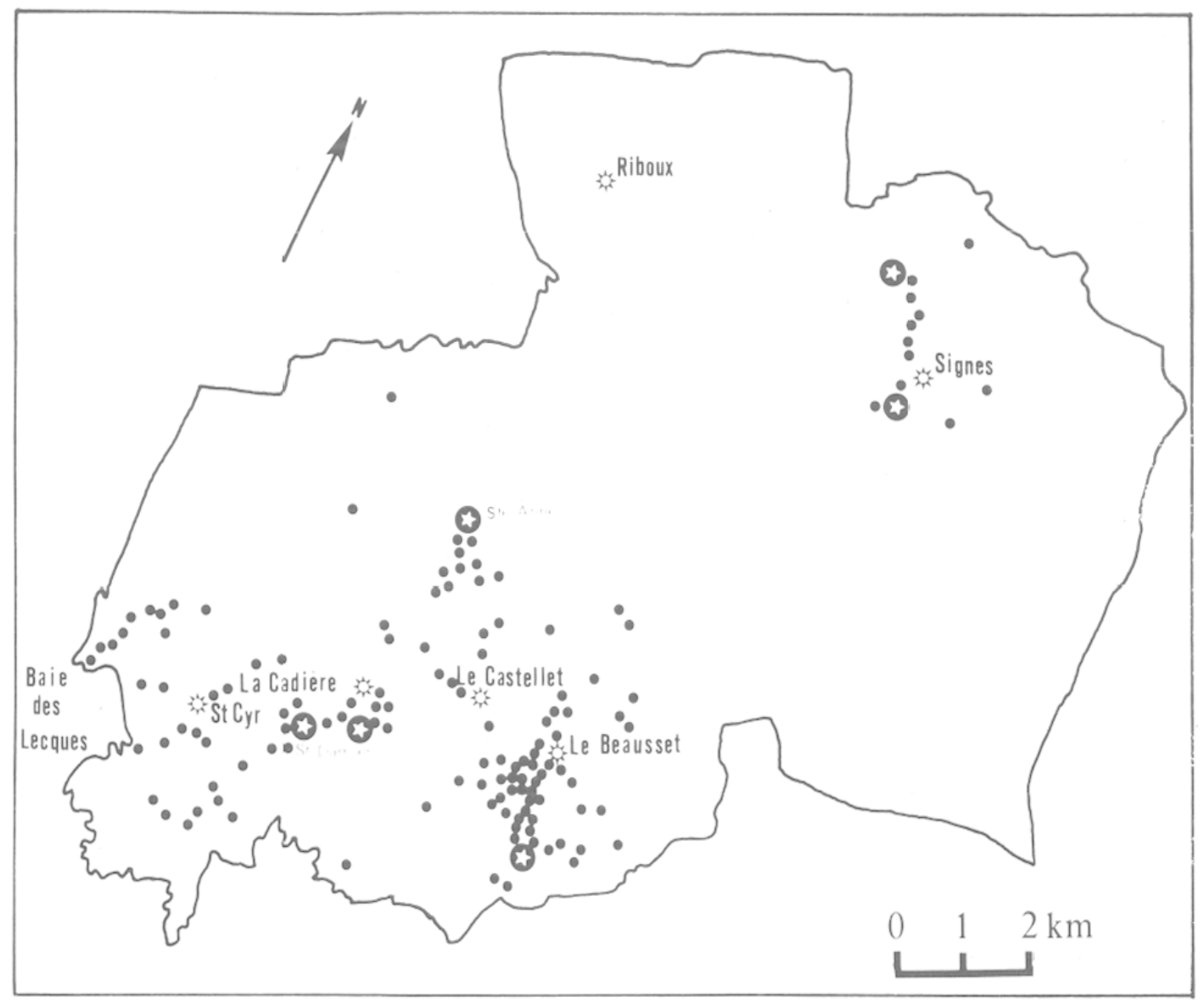

Carte $n^{\circ}$ 3. Les oratoires du canton du Beausset.

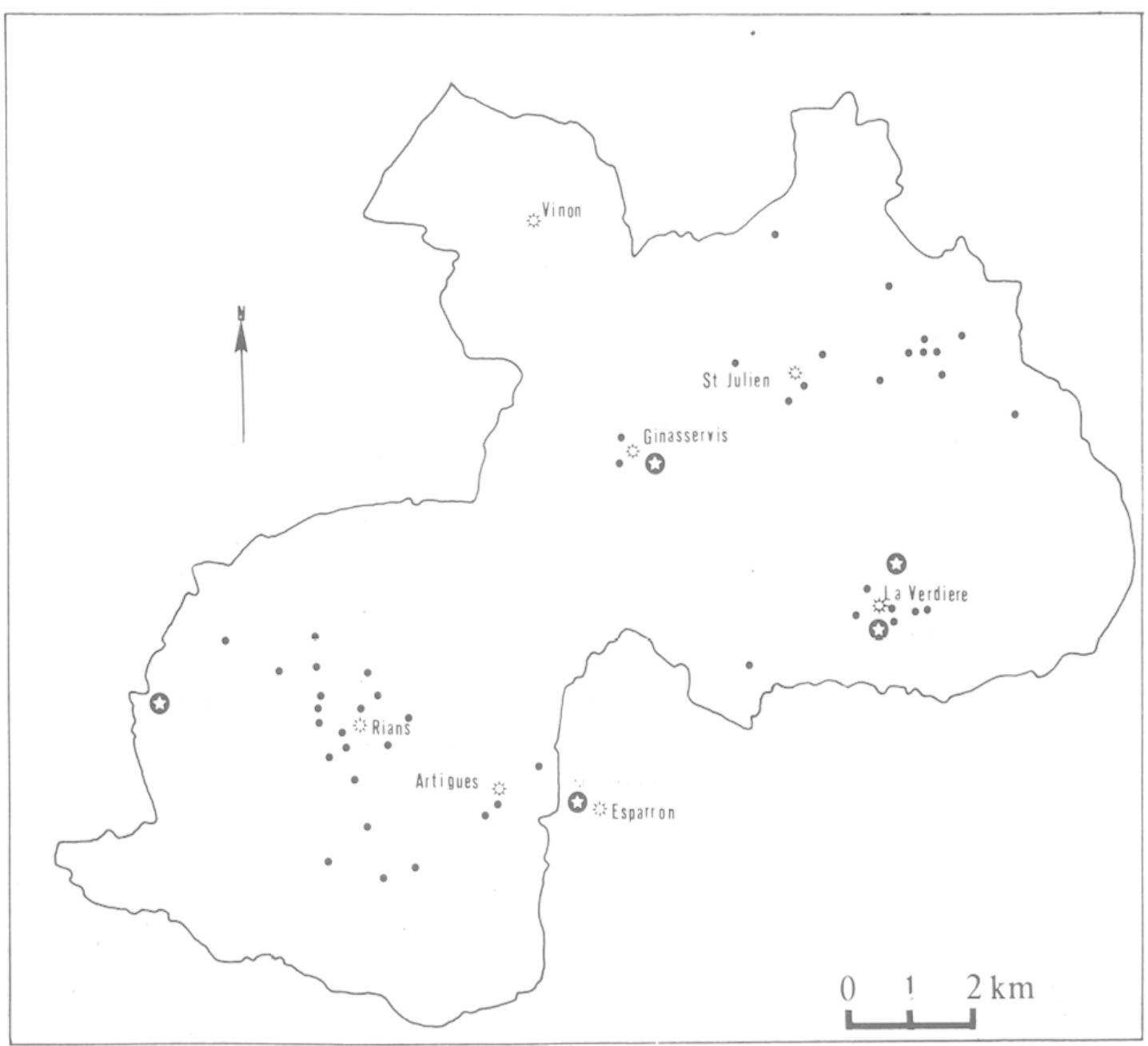

Carte $n^{\circ} 4$. Les oratoires $d u$ canton de Riuns.

- Oratoires

- Chapelles rurales

$*$ Villages 
plateau du Camp, indemne de toute urbanisation, et pour Rians au Bois du MontMajor, au plateau de Rians et, plus généralement, à tous les lieux peu habités et peu cultivés.

Les cent trente et un édicules du canton du Beausset se déploient pour la plupart dans le bassin du même nom, malgré l'isolement de onze d'entre eux dans le secteur nord-est correspondant au territoire de Signes. En partant de la mer (Baie des Lecques), on rencontre peu d'oratoires sur le littoral. La densité des monuments augmente aussitôt que l'on s'enfonce dans la vallée de Saint-Côme, région aux sols riches, d'occupation ancienne et exploitée dès l'abandon du bord de mer par les premiers habitants (19).

Continuons notre périple vers l'intérieur des terres. Nous constatons alors un partage du flux des oratoires. Certains se regroupent au nord-ouest, vers les communes de La Cadière et du Castellet ; la plupart se concentrent au sud-est, sur la commune du Beausset.

Les quarante-six édicules du canton de Rians se dispersent pour moitié dans la dépression qui traverse le canton d'est en ouest, quelques-uns s'alignant au sudest du chef-lieu dans la direction de Saint-Maximin. Au nord et au nord-est de Rians, le canevas des oratoires s'interrompt curieusement. Le plateau de Ginasservis. habité et pourvu de terres relativement bonnes, est quasiment vierge de monuments. Vinon, pour sa part, ne possède aucun édicule. On retrouve les oratoires dans la partie est du canton, regroupés autour du village de La Verdière et disséminés sur la commune de Saint-Julien le Montagnier. Rapprochons-nous dans l'espoir de comprendre cette distribution désordonnée.

Dans les deux cantons réunis, dix communes sur douze (20) possèdent des oratoires. A chaque chapelle rurale importante située dans une des communes du canton du Beausset correspond une concentration d'oratoires aux alentours ou, tout au moins, une diffusion plus grande de ces monuments. Le cas est flagrant dans la commune du Beausset, autout de l'ermitage de Notre-Dame du Beausset-Vieux (21). Même situation au Castellet et à La Cadière avec, respectivement, la chapelle Sainte-Anne et le Prieuré Saint-Damien, et surtout à Signes où la plupart des édicules jalonnent le chemin du sanctuaire de Notre-Dame l'Eloignée (22). Tout se passe comme si ces lieux de culte et de pèlerinages présidaient à la distribution d'unc grande partie des oratoires sur le terroir.

Dans les communes du canton de Rians, ce phénomène semble moins évident. La chapelle Saint-Estève, à l'ouest du chef-lieu, est totalement excentrée par rapport aux oratoires de la commune, et les édicules de Saint-Julien-le-Montagnier

(19) On sait que la Provence a trouvé dans les plaines et, en particulier, dans les plus petites, "ses pays neufs, ses Amériques intérieures" (Fernand BRAUDEL, La Méditerranée et le monde méditerranéen à l'époque de Philippe II, Paris, A. Colin, 1949, pp. 57 et 60). La vallée de Saint-Côme est un des plus anciens vignobles provençaux, la viticulture ayant été favorisée dans la contrée par la proximité des ports d'embarquement. De plus, le prieuré Saint-Damien, sis dans la vallée, figurera jusqu'à sa disparition, au milieu du XIV ${ }^{\mathrm{e}}$ siècle, en tête des meilleurs greniers à blé de l'abbaye Saint Victor de Marseille. Voir à ce sujet : Paul AMARGIER, "Un épisode de justice à La Cadière (Var) à la fin du X $X^{\mathrm{e}}$ siècle", Provence historique, XXVIII, 114, oct.-déc. 1978, p. 296. Voir également : Régine BROECKER, «Saint Damien et l'implantation victorine dans la région de La Cadière», Provence historique, XXXIII, 133, juilletaoût-sept. 1983, p. 337-338.

(20) Dans le canton du Beausset : Le Beausset (50 oratoires début 1984), Saint-Cyr (26), Le Castellet (25), La Cadière (19), Signes (11), aucun édicule à Riboux ; dans le canton de Rians : Rians (19), Saint-Julien-le-Montagnier (13), La Verdière (8), Artigues (4), Ginasservis (2), aucun oratoire à Vinonsur-Verdon.

(21) Carte $n^{\circ} 5$.

(22) Carte $^{\circ} 6$. 
sont dispersés sur un finage où l'on ne parvient pas à désigner un lieu privilégié de pèlerinage.

Les données historiques et ethnographiques permettent de nuancer ces premières impressions. Les communes du canton de Rians, à la différence de celles du Beausset, se caractérisent par une dévotion pérégrine davantage ouverte vers l'extérieur. De Rians, des pèlerinages partaient régulièrement vers Notre-Dame de Santé (La Verdière), Notre-Dame des Grâces (Cotignac), Notre-Dame des Oeufs (Gréoux-les Bains), la chapelle du Revest (Esparron) et même vers les îles de Lérins (23). De Saint-Julien-le-Montagnier, on allait à Notre-Dame des Grâces et Notre-Dame de Santé (24). De La Verdière, on gagnait Notre-Dame des Grâces et les chapelles Notre-Dame de Santé et Saint-Roch, toutes deux situées sur la commune (25). En revanche, la chapelle Saint-Estève semble être restée à l'écart des grands parcours pérégrins.

Il devient possible, dès lors, de proposer l'interprétation suivante. Comme au Beausset, la distribution d'une partie des oratoires rianssais s'organise en fonction de sanctuaires et de chapelles rurales mais, contrairement à ce canton, on ne peut l'appréhender à l'échelle communale ni même au seul niveau cantonal. Cette distribution s'effectue dans un espace géographique et social plus large. On ne saisit ce phénomène qu'en prenant en considération l'existence, dans les communes voisines, d'une chaîne d'oratoires qui comble les «blancs» de la carte cantonale. Ainsi, entre Artigues et La Verdière s'insère la commune d'Esparron et son patrimoine de neuf édicules. Plusieurs jalonnent les routes et les chemins qui s'étendent entre les deux premiers villages, tels ceux qui, à partir d'Esparron, rejoignent le sommet de la Montagne de l'Ouvière et la chapelle du Revest où venaient en procession les Rianssais et aussi, compte tenu de la proximité de ce lieu de culte, des habitants d'Artigues.

Si cette analyse est bonne, se mettrait alors en place de Rians à La Verdière en passant par Artigues et Esparron toute une théorie d'oratoires qui correspondrait à des parcours processionnels et à des cheminements vers des lieux de romérages. On soulignerait ainsi de nouveau, comme dans le canton du Beausset, le rôle des sanctuaires et de certaines chapelles rurales dans le «tissage» de la trame des oratoires. Une réelle étude topographique des 177 monuments érigés dans les deux cantons devrait, à cet égard, nous éclairer.

\section{Topographie des oratoires}

\section{Localisation des oratoires et des monuments païens}

Les trois quarts des édicules ( $74 \%$ ) s'élèvent au bord ou à proximité immédiate d'une route, d'un chemin public ou privé, ou d'un carrefour. Les monuments à l'écart d'une voie de circulation - isolés au milieu d'un champ, d'un bois, d'un

(23) Notice sur le pèlerinage de Rians à Lérins, 1864, 12 p. D'après le Cantique des pèlerins de Rians en l'honneur de Saint-Honorat, le trajet était le suivant : Aller : Rians, Notre-Dame des Grâces (Cotignac), Lorgues, Le Muy, l'Estérel, Cannes, Sainte-Marguerite. Retour : Draguignan, Salernes, Tavernes, Varages, Esparron, Artigues, Rians. De Forbin d'Oppède ajoute que la chapelle Notre-Dame de Santé de La Verdière était un lieu de passage pour les pèlerins qui se dirigeaient vers les îles de Lérins (DE FORBIN D'OPPĖDE, Monographie de la terre et du château de La Verdière, Marseille, Typographie et lithographie Marius Olive, 1880, p. 99).

(24) Abbé V. SAGLIETTO, Saint-Julien-le-Montagnier dans le Haut-Var, Toulon, Imprimerie du Sud-Est, 1943, p. 56.

(25) DE FORBIN D'OPPEDE, op. cit., p. 99. 
parc, ou perchés au sommet d'une colline - ne représentent que $26 \%$ du corpus.

En se fondant sur des observations semblables, de nombreux auteurs n'ont pu résister à la tentation de faire un rapprochement avec les monuments païens :

"L'oratoire se dresse à la rencontre des chemins, au "trivium" où, jadis, une statue de Mercure indiquait leur route aux voyageurs". (26).

La rapidité d'un tel saut dans le temps laisse perplexe. Certes, trivium ou quadrivium, les populations ont toujours considéré le carrefour comme un lieu magique (27) ou, le plus souvent, comme un endroit dangereux, hier parce que s'y rassemblaient les sorcières (28), les revenants, les jeteurs de sorts (29) et les démons (30), aujourd'hui parce qu'une bifurcation impose un choix à faire et évoque des risques objectifs (31). Néanmoins, l'identité de localisation entre les oratoires et les monuments païens n'est jamais rigoureusement démontrée. Si, au début de la christianisation, l'Eglise a trouvé quelque intérêt à utiliser des lieux de culte païens, le synode de Nantes, vers 658, impose leur destruction :

"Les arbres doivent être coupés, les racines arrachées et brûlées, les pierres enlevées et jetées en un autre lieu, là où elles ne pourront plus servir au culte» (32).

L'Eglise a eu alors le choix entre deux comportements opposés : continuer à occuper l'ancien site dans l'espoir de parvenir à contrôler la dévotion des fidèles (33) ou changer de lieu de culte en escomptant favoriser ainsi l'oubli du premier. On peut imaginer les deux attitudes et, dans le doute, rien n'autorise les généralisations fréquentes consistant à confondre la localisation des édifices religieux d'aujour-

(26) Louis PORCHERON, op. cit., p. 411. Dans ce registre les auteurs font chorus. Voir par exemple : Robert BAILLY, Les chapelles rurales en Provence, Avignon, F. Orta, 1969, p. 9 ; G. CHARRIËE. "Feux, bûchers et autodafés bien de chez nous", Revue de l'histoire des religions, t. CXCIV-1, Juillet 1978, p. 24 ; Alphonse CHIDE, «Les oratoires de Provence», La revue des pays d'Oc, avril-juin 1933, p. 218 ; Albert DAUZAT, Le village et le paysan de France, Paris, Gallimard, 1941, p. 146 ; Pierre IRIGOIN, Les oratoires de Provence, Aix-en-Provence, Amis des oratoires, 1940, p. 11 ; René JOLIN, Inl''ntaire des chapelles de Pierre Bleue du Hainaut, Metz, Est Imprimerie, 1972, p. 9 ; Gabriel LE BRAS, l. église et le village. Paris, Flammarion, 1976, p. 102 ; P. SAINTYVES, En marge de la légende dorée, Paris, Librairie Critique Émile Nourry, 1930, pp. 267-268 ; etc.

(27) "Jadis en Basse-Provence, aux carrefours, les paysannes faisaient bénir les "simples": la bourrache qui fait suer, l'hysope qui arrête les palpitations, la sauge pour la digestion, le thym pour le mal de ventre, etc.» (Claude SEIGNOLLE, Le Folklore de la Provence, Paris, G.P. Maisonneuve et Larose, 1963, p. 192).

(28) Marc AUGÉ, Génie du paganisme, Paris, Gallimard, 1982, p. 220.

(29) Claude LECOUTEUX, "Paganisme, christianisme et merveilleux", Annales ESC, juillet-août $1982, \mathrm{n}^{\circ} 4, \mathrm{p} .707$.

(30) Marcel MAUSS, «Esquisse d'une théorie générale de la magie», Sociologie et anthropologie, Paris, PUF, 1950, p. 39 ; Hervé MARTIN, Louis MARTIN, "Croix rurales et sacralisation de l'espace. Le cas de la Bretagne au Moyen-Age», Archives de Sciences Sociales des Religions, janvier-mars 1977, $43 / 1$, p. 29.

(31) Ainsi, à La Garde (Var), les paroissiens érigent en 1954 un oratoire dédié à Notre-Dame de la Route à l'intersection de cinq voies à forte circulation.

(32) Claude LECOUTEUX, op. cit., p. 705. Rappelons par ailleurs que les monuments mégalithiques des Ligures furent détruits eux aussi, en particulier lors du défrichement gallo-romain (ESCALON DE FONTON in Edouard BARATIER, Histoire de la Provence, Toulouse, Privat, 1969, p. 30).

(33) Une partie du clergé local a revendiqué ce comportement : "Qui sait même si la religion n'avait pas voulu, en émaillant de chapelles rustiques cette terre imprégnée des cendres des Grecs et des Romains, effacer le culte des divinités topiques". Abbé Magloire GIRAUD, Archives puroissiales ou statistique religieuse de La Cadière, Toulon, Imprimerie d'E. Aurel, 1858, p. 30. 
d'hui avec celle des cippes ou des laraires de jadis (34).

Il existe une autre éventualité. La construction des oratoires ne serait-elle pas un effet du combat du clergé post-tridentin ? Erigés dans l'espace rural, c'est-à-dire généralement assez loin de l'église paroissiale, ces monuments pourraient être l'asile des saints expulsés de l'église, évincés par certains clercs à cause des relents de polythéisme (35) qu'évoque la dévotion dont ces intercesseurs sont l'objet (36). Cette hypothèse d'une survivance du paganisme offre de nouveaux arguments à ceux qui voient une filiation entre le culte des oratoires et celui des monuments païens. Mais là encore, aussi intéressante que soit cette perspective, nulle preuve, nulle démonstration convaincante ne permet d'établir cette filiation avec certitude, tout au moins pour ce qui concerne la localisation des différents monuments.

\section{Localisation des oratoires dans l'espace rural}

Quatre-vingt-onze pour cent des monuments des deux cantons s'élèvent à l'extérieur des agglomérations. L'oratoire est un monument essentiellement rural. Encore doit-on apprécier ces chiffres à la lumière du processus d'urbanisation intense connue par la région depuis deux décennies. Dans certaines communes (Le Beausset, Saint-Cyr, La Cadière), la croissance du bâti villageois a absorbé des oratoires situés autrefois hors les murs.

A l'intérieur de l'espace rural, une distinction s'impose entre, d'une part, les édicules érigés dans l'ager, c'est-à-dire dans ou à proximité des champs, ou dans les guérets, ou bien encore sur des terres cultivées récemment abandonnées et, d'autre part, les monuments édifiés dans le saltus (37). Le premier groupe représente $71 \%$ des oratoires ruraux. Les autres édicules $(29 \%)$ se dressent dans les bois. Ici encore, il faut évaluer ces chiffres en se référant à la progression régulière du saltus aux dépens de l'ager : dans le Var, la surface boisée a doublé en l'espace

(34) La situation d un oratoire à proximité du tracé d'une ancienne voie romaine ne prouve pas davantage la réalité d'une survivance, sauf si la présence antérieure d'un monument païen sur cette même voie est attestée. Dans un autre domaine, la présomption de relations entre le culte des sources et celui des oratoires, qui apparaît comme un leitmotiv dans les ouvrages spécialisés, semble peu fondée dans la région étudiée. Deux fois seulement, nous avons constaté l'érection d'un édicule près d'une source, à Saint-Julien-le-Montagnier avec l'oratoire Saint-Joseph et au Beausset avec l'oratoire Notre-Dame de Lourdes. Dans l'Inventaire des oratoires du Var de M. L. JANVIER (op. cit.), on ne compte pas plus de 7 édicules dans le même cas.

(35) A Aix-en-Provence, l'oratoire Saint-Mitre a pendant longtemps été l'objet d'une méprise : la statue du saint vénéré par les fidèles représentait en réalité le dieu Priape ! Pierre IRIGOIN, Les oratoires de France depuis les origines, Aix-en-Provence, Amis des oratoires, 1977, p. 22.

(36) Face à un clergé qui veut «enfermer et circonscrire le "sacré" dans des lieux précis" (MarieHélène FROESCHLEE-CHOPARD, La religion populaire en Provence orientale au XVIII siècle, Paris, éd. Beauchesne, 1980 , p. 345), les oratoires constituent une sorte de défi. On comprend alors le regroupement fréquent des chapelles rurales, des ex-voto et de nombreux édicules dans un même lieu, destination des romérages et repaire de toutes les pratiques rejetées par la norme ecclésiale. Au XVIII ${ }^{e}$ siècle en particulier, l'épiscopat provençal va lutter contre les chapelles et les oratoires. Voir Michel VOVELLE in Edouard BARATIER, op. cit., p. 362.

(37) «Le saltus représente l'ensemble des terrains qui ne sont pas régulièrement cultivés et qui n'ont pas de couvert forestier continu et fermé». (Georges BERTRAND, "Pour une histoire écologique de la France rurale", in Georges DUBY, Armand WALLON, Histoire de la France rurale, Paris, Seuil, 1975, t. I, p. 80). Par leurs caractères (discontinuité, absence de densité), les bois des deux cantons s'apparentent davantage au saltus qu'à la silva. Précisons que pour localiser un oratoire dans l'ager ou le saltus, nous n'avons pas considéré le micro-site - "parcelle de terrain sur laquelle est construit le monument" (Nicole NIVELLE, Code pour l'analyse des monuments religieux, Paris, éd. CNRS, 1975, t. I, p. 36) -, mais l'environnement immédiat. 
d'un siècle (38). Des oratoires jadis dans l'ager appartiennent aujourd'hui au saltus.

\section{Périodisation}

\section{Oratoires anciens et récents}

Le faible rendement de cette première approche topographique tient à une lacune de taille : l'absence d'une perspective diachronique, pourtant indispensable à quiconque veut étudier un objet ou un monument cultuel (39).

L'oratoire est un monument difficile à dater. S'engager dans une telle entreprise revient à s'exposer à bien des déconvenues. A partir des comptages effectués dans l'Inventaire des oratoires $d u$ Var de M. Louis Janvier (cf. note 15), nous avons recensé 108 oratoires érigés à une date inconnue et 305 monuments, anciens d'après le contexte, mais inclassables avec certitude dans un siècle ou dans un autre. C'est dire combien les données sont lacunaires : $49 \%$ des huit cent trente-huit oratoires varois ne trouvent pas de place dans une périodisation séculaire !

L'enquête de terrain et le fichier des Amis des oratoires (40) permettent heureusement d'enrichir partiellement ces données décevantes. D'une part, on parvient presque toujours à obtenir la date exacte de l'érection des édicules construits à une époque récente. D'autre part, l'enquête orale lève parfois l'incertitude qui entoure les autres monuments. Toutefois, les renseignements recueillis pour les oratoires vieux de plus d'une cinquantaine d'années manquent le plus souvent de précision, les villageois se contentant de les qualifier d' "anciens".

Nous avons alors décidé de distinguer dans les deux cantons, d'une part des oratoires anciens, d'autre part des oratoires récents, en prenant 1955 comme date de référence.

Pourquoi 1955 ? Au cours de recherches antérieures conduites dans la même région, nous avions pu montrer que le milieu des années 1950 s'était caractérisé par une accentuation du mouvement de déruralisation : début des ventes massives de terres agricoles au profit de la fonction résidentielle, augmentation du taux moyen d'abandon annuel des cultures, progression fulgurante des demandes de permis de construire, essor démographique dû à un apport extérieur, baisse de la population active agricole (41). L'ère du «tourisme diluvien» (42) commençait alors.

(38) I.D.R.E.E.S. (Institut de Développement Régional Économique, Écologique et Social), «La Forêt varoise. Diagnostics et propositions. Indications pour une politique de la forêt méditerranéenne", Cahiers de la Méditerranée, juin 1981, p. 55.

(39) Bernard COUSIN, «Ex-voto. Document d'histoire, expression d'une société», Archives des Sciences Sociales des religions, 1979, 48.1, p. 115. Voir également Marie-Hélène FROESCHLÉ-CHOPARD, "Pour une cartographie des lieux de culte», Revue d'histoire de l'Église de France, $\mathrm{t}$. LXV, $\mathrm{n}^{\circ}$ 175, juil.-déc. 1979, p. 302. Sur la confusion entre fréquence monumentale et fréquence de la dévotion qu'entraîne l'absence de périodisation, voir VAN GENNEP, op. cit., p. 123.

(40) Chaque fiche est souvent accompagnée de photographies ou d'articles de presse qui permettent de préciser la date d'érection ou de restauration de l'édicule.

(41) Les deux cantons, à des degrés différents, sont touchés par la déruralisation : alors qu'en 1954 les actifs agricoles (exploitants et salariés) représentaient dans les cantons du Beausset et de Rians respectivement $51,7 \%$ et $63,5 \%$ de la population active, leur part chute à $16 \%$ et à $20,3 \%$ en 1975 . On perçoit ici l'ambiguiité de l'usage du terme rural, jamais clairement défini, lors d'enquêtes dans des collectivités où ne cesse de crôttre la diversification des agents économiques. Cette remarque n'a rien de gratuit, car la notion d'espace rural peut véhiculer, aux yeux du chercheur comme à ceux du lecteur, tout un ensemble de représentations laissant croire qu'il existe encore obligatoirement une société rurale là où on parle d'espace rural. Il n'est par conséquent pas inutile de préciser que l'utilisation faite ici de cette notion ne préjuge en rien de la «ruralité» des sociétés considérées.

(42) Michel MARIE, Un territoire sans nom. Pour une approche des sociétés locales, Paris, Librairie des Méridiens, 1982, p. 39. Dans le domaine religieux, les années 1950 correspondent par ailleurs à une époque de remise en question du dogme de l'Église (prêtres ouvriers, catholicisme social, etc.) qui précède de quelques années le XXI ${ }^{\mathrm{e}}$. Concile oecuménique de Vatican II. 
L'oratoire étant, nous l'avons vu, un monument essentiellement rural - et de ce fait lié peu ou prou, semble-t-il, à la vie rurale -, il nous a paru légitime d'effectuer la bipartition du corpus en considérant l'époque où s'est accru le processus de déstructuration de la société rurale traditionnelle. Ce choix, avouons-le, imposé par la nature même des sources, n'est pas rigoureusement fondé méthodologiquement. Le déroulement et les résultats de la recherche lui ont néanmoins donné un certain degré de pertinence.

\section{Nouvelle approche topographique}

Dans les deux cantons réunis, nous avons recensé 112 monuments anciens et 65 édicules récents (43). Contrairement à de nombreux préjugés nourris par les théories de la survivance, on ne peut faire des oratoires les vestiges d'une dévotion du passé : dans le canton du Beausset en particulier, où des édicules furent encore érigés en 1984 (44), aucun tarissement des fondations n'est perceptible.

\begin{tabular}{|l|c|c|c|c|}
\hline \multicolumn{1}{|c|}{ CANTONS } & $1955-1964$ & $1965-1974$ & $1975-1984$ & TOTAL \\
\hline Le Beausset & $11(18 \%)$ & $30(50 \%)$ & $19(32 \%)$ & $60(100 \%)$ \\
\hline Rians & $4(80 \%)$ & $1(20 \%)$ & - & $5(100 \%)$ \\
\hline
\end{tabular}

Les constructions d'oratoires récents dans les cantons du Beausset et de Rians.

La répartition des monuments anciens et récents entre l'espace rural et l'espace urbain ne fait pas apparaître de bouleversement sensible : on trouve une proportion voisine d'oratoires ruraux (ou urbains) dans l'ensemble des édicules récents comme dans celui des édicules anciens.

Les pourcentages entre ager et saltus, en revanche, connaissent un léger glissement. La part des oratoires récents et ruraux présents dans l'ager (83\%) l'emporte sur celle des édicules anciens et ruraux dans le même cas $(64 \%)$. La relative forte proportion $(36 \%)$ de monuments anciens dans le saltus peut s'expliquer, au moins partiellement, par la progression de la surface boisée. Seuls les oratoires anciens, en effet, ont pu être vraiment touchés par ce phénomène. Mais on peut songer à une autre explication.

Il convient de se garder d'un regard anachronique consistant à concevoir l'opposition ager/saltus jadis dans les mêmes termes qu'aujourd'hui. Du point de vue du système de production, cette opposition se justifiait beaucoup moins naguère lorsque l'agriculture occupait une position dominante - que de nos jours où l'on perçoit le saltus essentiellement comme un espace improductif, même si la chasse ou la cueillette des champignons ont pu rester des "passions" pour les Varois.

Les bois sont demeurés longtemps le lieu de pratiques productives, en particulier dans le Haut et le Moyen Var, qui, loin d'être accessoires, représentaient un complément des activités rurales (45). Le saltus constitutait alors «une annexe, par-

(43) Ces chiffres doivent être appréciés en tenant compte du fait que les oratoires anciens constituent sans nul doute un corpus résiduel, de nombreux monuments ayant aujourd'hui disparu à la suite de destructions ou de dégradations.

(44) En septembre 1984 , un $51^{\text {e }}$ oratoire a été édifié sur la commune du Beausset. La part des oratoires récents dans le total des monuments est différente selon les cantons : $46 \%$ au Beausset et $11 \%$ seulement à Rians.

(45) Roger LIVET, Habitat rural et structures agraires en Basse-Provence, Aix-en-Provence, Ophrys, 1962, p. 386. 

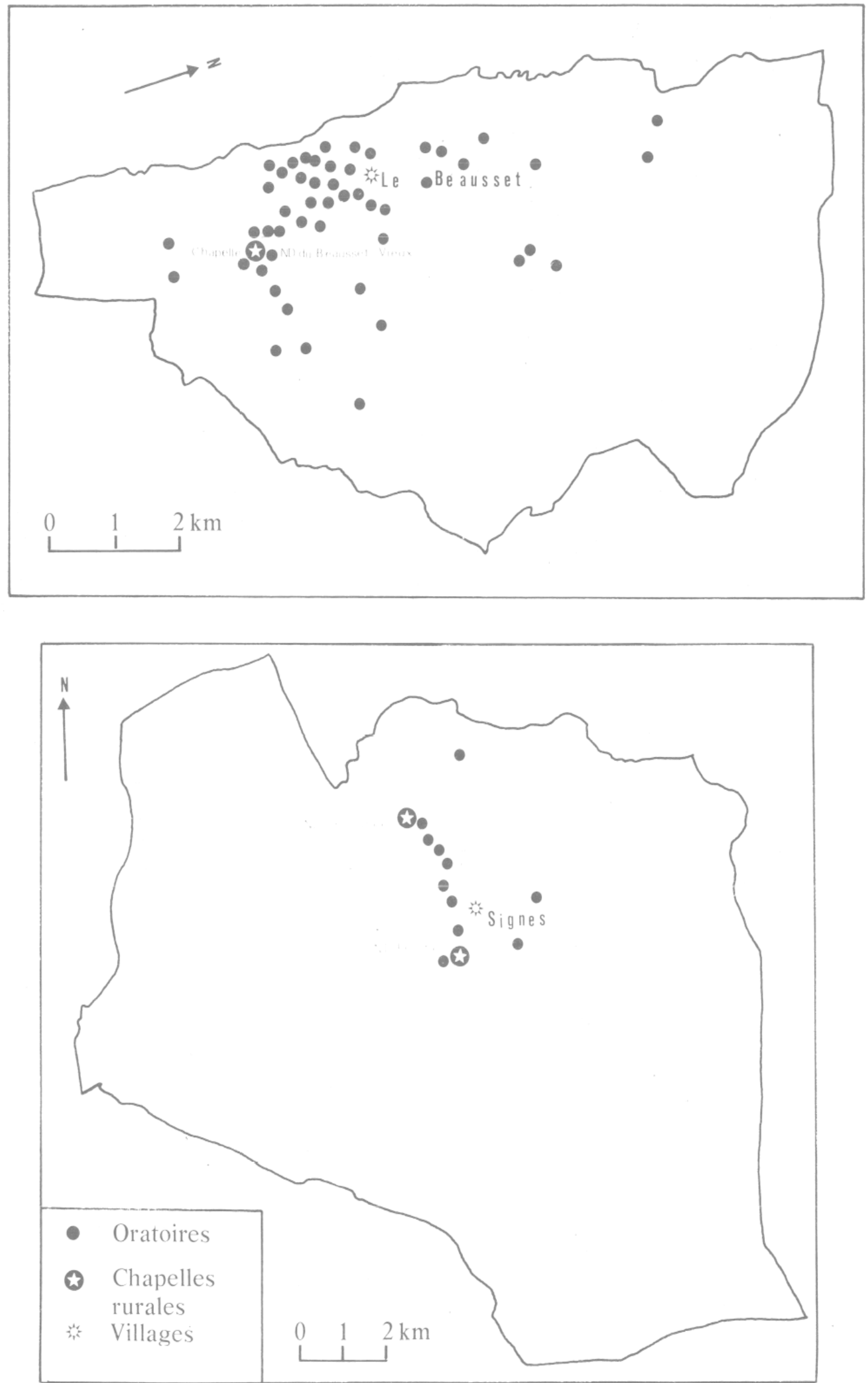

Carte $n^{\circ} .5$ et 6. Les oratoires de la commune du Beausset et de la commune de Signes. 
fois indispensable, de l'espace agricole qui l'inclut" (46). Si les oratoires anciens sont plus nombreux dans le saltus que les édicules récents, c'est donc, probablement, parce qu'il s'agissait autrefois d'un espace occupé et parcouru quotidiennement. De ce fait, le saltus n'avait rien de sauvage.

La situation des édicules par rapport aux routes, chemins et carrefours s'éclaire d'un jour différent. Si, rappelons-le, $74 \%$ de l'ensemble des oratoires se situent à proximité immédiate d'une voie de communication, la part des monuments anciens est de $58 \%$, et celle des édicules récents de $16 \%$. Autrement dit, $45 \%$ des oratoires récents s'élèvent à proximité d'une voie de passage contre $91 \%$ des oratoires anciens.

Nous aurions pu prévoir cela si, au moment de la première localisation des monuments, nous avions distingué Le Beausset et Rians. Dans le second canton, où dominent les oratoires anciens, $89 \%$ des monuments sont proches d'un chemin ou d'une route, contre $69 \%$ au Beausset où les oratoires récents représentent une partie importante du corpus. De plus si, dans les deux cantons réunis, on différencie les monuments situés sur des chemins privés et ceux qui jalonnent les chemins (ou routes) publics, on constate alors que $3 \%$ seulement des oratoires anciens érigés à proximité d'une voie de communication entrent dans la première catégorie, contre $66 \%$ des édicules récents.

Si nous insistons sur ce point, c'est parce qu'il suggère une accessibilité différenciée des oratoires. Les monuments érigés sur un chemin ou une route publics ou à leurs abords immédiats - offrent, par ce fait même, un accès facile, qu'ils soient dans un espace public ou privé. Les autres édicules sont au bord de chemins privés à l'intérieur des propriétés ou, le plus souvent, dans des parcs enćlos.

\begin{tabular}{|c|c|c|c|c|c|c|}
\hline Cantons & Datation & $\begin{array}{l}\text { Chemins } \\
\text { publics }\end{array}$ & $\begin{array}{l}\text { Chemins } \\
\text { privés }\end{array}$ & $\begin{array}{l}\text { Sites } \\
\text { privés } \\
\text { (1) }\end{array}$ & $\begin{array}{l}\text { Sites } \\
\text { publics } \\
\text { (2) }\end{array}$ & Total \\
\hline \multirow{2}{*}{ Le Beausset } & Anciens & 60 & 3 & 7 & 1 & 71 \\
\hline & Récents & 8 & 19 & 33 & - & 60 \\
\hline \multirow{2}{*}{ Rians } & Anciens & 39 & & 1 & 1 & 41 \\
\hline & Récents & 2 & - & 3 & & 5 \\
\hline \multirow{3}{*}{ Total } & Anciens & 99 & 3 & 8 & 2 & 112 \\
\hline & Récents & 10 & 19 & 36 & - & 65 \\
\hline & $\begin{array}{c}\text { Anciens } \\
+ \\
\text { récents }\end{array}$ & 109 & 22 & 44 & 2 & 177 \\
\hline
\end{tabular}

Accessibilité des oratoires. (1) et (2) : sites autres que des voies de communication (parcs, sommets de collines, etc.)

(46) Yves RINAUDO, "Note sur l'extension de la forêt varoise au XIX ${ }^{\mathrm{e}}$ siècle», Forêt méditerranéenne, t. 2, $n^{\circ} 2$, mars 1980 , p. 175 . En 1839 , les droits d'usage grevaient $48 \%$ de la superficie forestière du Var. Rappelons l'importance des bois pour la quête du combustible (alimentation des fours banaux, des forges, des fours domestiques, des fours à cade, fascines pour les chaufourniers), la pratique du soutrage, le travail des "pegouliè» (collecteurs de résine destinée à la fabrication de poix pour la fabrication navale), des charbonniers et de tous les «bouscatié", l'usage de la charrée, le ramassage des plantes les plus diverses (aromatiques, tinctoriales, médicinales ou alimentaires), le parcours des troupeaux, etc. 
Il est alors légitime d'introduire le critère de la privatisation : les oratoires "privatisés" sont ceux auxquels tout un chacun ne peut pas (ou très difficilement) avoir accès. Inversement, on propose d'appeler "publics" les édicules aisément accessibles, même lorsqu'ils appartiennent à des personnes privées. On constate alors que si les oratoires "publics" (chemins et sites publics) représentent $63 \%$ des cent soixante-dix-sept monuments, les pourcentages changent lorsqu'on distingue au sein du corpus les édicules anciens et récents : $90 \%$ des premiers sont «publics" contre seulement $15 \%$ des seconds. C'est dire que la tendance à la privatisation des oratoires récents se dessine très clairement.

\section{De profondes mutations}

Avant de dégager les conséquences de cette évolution, il convient de mettre en exergue un des intérêts majeurs de ces édicules : ils rendent possible un "commerce familier» avec les saints.

Sur le terrain, les difficultés rencontrées dès l'instant où l'on s inquiétait de la date précise d'un monument ancien n'ont jamais laissé de nous étonner : «Il a toujours été là !», telle était la réponse commune. Cette notion d'un temps immémorial ne semble pas singulariser la région étudiée (47). Les autochtones parlent de l'oratoire ancien comme d'un monument intégré depuis toujours à leur environnement (48). L'édicule a toutes les apparences d'un monument banal, auquel, ordinairement, on ne prête pas une grande attention, mais où, à tout moment, on sait pouvoir trouver le titulaire, sans peine aucune compte tenu de sa proximité.

Ce voisinage immédiat de l'oratoirc - ancien ou récent - autorise une multiplication des médiations avec le saint. Se placer sous l'égide du titulaire permet aux autochtones d'avoir leur propre "gestion du sacré" (49), privilège qui présente au moins trois avantages.

D'abord, on peut ainsi s'adresser directement au saint sans passer par l'intermédiaire d'un clerc, souvent soupçonné de faire le tri entre les demandes recevables et celles qui, à ses yeux, le sont moins. Ensuite, l'oratoire et son titulaire offrent la possibilité d'une dévotion "à bon compte» lorsque les circonstances, les travaux des champs, l'éloignement (50), rendent contraignant le déplacement jusqu'à l'église ou au sanctuaire de la commune (51). Enfin, dernier avantage, la proximité physique d'un saint représenté par une petite statue manipulable (52), abrité dans

(47) Voir par exemple Louis BRUN, Orutoires de nos terroirs : cantons de Bourg-Saint-Andéol (Ardèche). Pont Saint-Esprit, Bagnols-sur-Cèze (Gard). Nîmes, Louis Brun. 1976, p. 41.

(48) En réalité s'opposent aux villageois pour qui l'oratoire "a toujours été là" ceux pour qui il n’a jamais été là, c'est-à-dire ceux qui ne voient pas les édicules. Ceci semble être une attitude nouvelle.

(49) Expression empruntée à Pierre BOURDIEU, "Genèse et structure du champ religieux", Revue de Sociologie. XII, 1971, p. 308.

(50) Voir Paul RAYBAUD. Michel PERRÉARD, L architecture rurale française. Corpus des genres. des types et des variantes. Comté de Nice. Paris, Berger-Levrault, 1982, p. 195 : l'oratoire Notre-Dame étudié dans cet ouvrage fut édifié à la fin du XIX" siècle, "à l'initiative du propriétaire des lieux, pour satisfaire aux voeux de ses métayers qui, pour des raisons de travail et d'éloignement du village. ne pouvaient pas, tous ensemble, assister à la messe du dimanche. Par roulement, les membres de la communauté (une vingtaine de personnes) étaient dispensés, par le curé. de l'obligation dominicale, à la condition que ceux demeurés à la propriété récitassent un chapelet devant l'oratoire».

(51) A Saint-Julien-le-Montagnier, le prêtre est obligé d’insister auprès de l’évêque afin d’obtenir l'autorisation du binage lors de certaines fêtes, à cause "de l'impossibilité pour la plupart de nos paysans d'abandonner tous à la fois la ferme pour assister à une scule messe». Coutumier pour la paroisse de Saint-Julien de 1884. Archives diocésaines de Fréjus-Toulon.

(52) Sur ce point, voir Alban BENSA. Les saints guérisseurs du Perche-Gouët. Espace symbolique du Bocage. Paris, Institut d'Ethnologie, 1978, p. 173. 
un monument de taille humaine, facilite le commerce "familier" avec l'intercesseur, au double sens de ce mot puisque, parfois, le saint est presque considéré comme un membre de la famille (53), doté d'un pouvoir personnel. Beaucoup plus disponible qu'un Dieu abstrait et inaccessible, on peut aisément entrer en contact avec lui, lui parler, le réprimander, éventuellement le toucher - comportements d'autant plus difficiles à adopter dans une église que le clergé, par toute une organisation adéquate de son espace intérieur, cherche, dans bien des cas, à maintenir des distances dissuasives (et respectueuses) entre les objets de dévotion (statues perchées, autels inaccessibles) et les fidèles.

Dans le cas des oratoires anciens n'importe pas seulement la proximité physique de l'intercesseur et du fondateur mais aussi celle du saint et du champ. En pleine campagne, ces édicules

«semblent bénir les cultures, éloigner la foudre ou la grêle. Ils se dressent dévotement au milieu des vignes et des oliviers ; parfois, sur un tertre, ils dominent la campagne. Le jour des Rogations, le clergé, suivi des fidèles, monte en procession, s'agenouille et supplie Saint Marc ou Saint Eloi de protéger les récoltes, de garder les troupeaux" (54).

Processions et Rogations : la distribution de nombreux oratoires autour des sanctuaires, évoquée précédemment, suggérait l'existence de chemins de dévotion. Dans la plupart des localités étudiées, une grande partie des édicules peut être mise en relation avec des pèlerinages, des processions ou des pratiques cérémonielles variées. Ici, ne sont pas seuls pris en considération les monuments qui jalonnent les sentiers des chapelles rurales et des ermitages (55) mais aussi ceux qui, d'une manière ou d'une autre, marquaient jadis le parcours ou le terme d'une procession où, parfois, le profane le disputait au religieux. A Saint-Cyr, le jour de la fête de la Saint Jean, les villageois allaient en groupe allumer le feu de joie traditionnel près de l'oratoire dédié à ce saint, érigé sur l'emplacement d'un ancien "farot", au sommet de la colline du Peyroulet. Cette pratique a perduré jusqu'au début du $\mathrm{XX}^{\mathrm{e}}$ siècle. Dans la même commune, on organisait pour la Saint Jules une fête autour de l'oratoire dont cet intercesseur est le titulaire, à laquelle participaient les habitants des quartiers environnants. A Artigues, s'en allaient en procession vers "l'oratoire de la montagne» les familles demeurant dans les quartiers proches de l'édicule Sainte-Foy. Les autochtones partaient aussi de l'oratoire Saint Pierre et Saint Roch pour monter à genoux jusqu'au sommet de la colline voisine. A Ginasservis, pour la Saint-Eloi, les fidèles se dirigeaient en procession vers l'oratoire du saint (aujourd'hui disparu) où l'on bénissait les bêtes (56). A La Verdière, on associait la bénédiction de l'oratoire Sainte-Magdeleine avec la bravade. Au Beausset, des cavalcades avaient lieu devant l'oratoire Saint-Eloi il y a encore une vingtaine d'années. A Saint-Julien-le-Montagnier :

"le dimanche après Saint Jean les prieurs portent à l'autel Saint Eloi et le pain pour les fidèles et celui réservé aux animaux. Le prêtre le bénit à

(53) La prière suivante, récitée à Brillac (Charentes) jusqu'à l'entre-deux guerres, est à cet égard très significative : «Le Bon Dieu est mon père, la Vierge ma mère, les Saints sont mes frères, les Saintes mes soeurs». M. LEPROUX, Dévotions et Saints guérisseurs, Paris, PUF, 1957, p. 5.

(54) Louis PORCHERON, op. cit., p. 412.

(55) Sentiers souvent appelés "chemins des oratoires".

(56) Coutumier de Ginasservis (sans date), Archives diocésaines de Fréjus-Toulon. 
la première messe. Après la grand messe qui se dit à neuf heures pour faciliter les cavaliers le prêtre déjeune et avec les porteurs du saint et les clercs descend au cours, bénit les bêtes, fait la procession à l'oratoire près la remise, retourne avec le tambour en tête à l'église» (57).

Dans le hameau des Rouvières, qui fait partie de la commune de Saint-Julien, les quatre principales processions (Saint-Nom de Jésus, Saint-Eloi, Saint-Joseph, Sainte-Philomène), correspondent à des titulatures d'édicules anciens (58). Les habitants du hameau se rendaient jadis au pied de l'oratoire Sainte-Philomène le jour de la fête de la sainte.

Dans d'autres communes, nous pourrions aussi bien appeler oratoire processionnel, ou cérémoniel, celui devant lequel les convois funèbres marquaient un arrêt. comme Saint-Honorat à Esparron ou Saint-Louis d'Anjou à Brignoles.

La première qualité d'un monument processionnel est d'être l'objet de formes de dévotion collectives et organisées. Les Rogations représentaient un moment privilégié de ce type de culte. "A tous ces oratoires, on faisait des reposoirs", se souvient une habitante de Saint-Julien-le-Montagnier. Ces cérémonies, qui ont fortement marqué la tradition orale (59), étaient par conséquent des processions à stations (60) qui faisaient du parcours du finage une succession de requêtes auprès des saints.

L'oratoire processionnel permettait cette station en différents endroits du terroir. lieux de cérémonies propitiatoires ou gratulatoires $s$ 'inscrivant dans le cadre de la conjuration du malheur biologique ou écologique.

On compte 39 oratoires processionnels dans le canton du Beausset et 20 dans celui de Rians. Or. parmi ces 59 monuments, un seul appartient au groupe des édicules récents. Ceci démontre incontestablement que tout a changé avec la privatisation de cette catégorie d'oratoires. Situés dans un espacc privé, ils nc pcuvent plus faire l'objet de cérémonies religieuses périodiques entraînant toute la communauté. La privatisation des monuments révèle une mutation dans le commerce familier avec les saints. Elle s'accompagne d'une privatisation de la dévotion, caractérisée par un repli sur le groupe familial (61), contrairement à l'oratoire ancien qui était l'objet d'une dévotion publique. Dans cette perspective du passage du public au privé il est d'ailleurs remarquable que les édicules récents servent souvent de

(57) Coutumier pour la paroisse de Saint-Julien de 1884. Archives diocésaines de Fréjus-Toulon.

(58) Permissions pour la paroisse des Rouvières. Archives diocésaines de Fréjus-Toulon.

(59) Et aussi littéraire : les processions qui, à partir des trois villages de Cuges. du Castellet et de Sainte-Anne du Castellet se dirigeaient vers l'oratoire dédié à Saint-Antoine. Saint-Clair et Sainte-Anne. chacun étant le patron des localités, ont fourni l'argument principal d'un roman remarquable dans la mesure où l'auteur a su systématiser tout ce qui participe, en ce domaine, du processus de légendification des pratiques cérémonielles : compétition dans les attitudes ostentatoires entre villages lors des Rogations, débordements festifs et contrôle des fidèles par les Pénitents, érection de l'oratoire après avoir obtenu des pluies bienfaisantes à la suite d'une période de sécheresse exceptionnelle, etc. (Nicole CIRAVEGNA, Les trois jours du cavalier. Paris, Seuil, 1979, 236 p.).

(60) La plupart des ouvrages sur les oratoires mentionnent les stations devant les monuments lors des Rogations. Voir par exemple : Jean GAVOT, Les oratoires bas-alpins, Aix-en-Provence. Amis des oratoires, sans date, p. 8 ; Louis HENSELING, "Les oratoires du Var», Bulletin de la Société des Amis $d u$ Vieux Toulon, janv.-fév.-mars 1936, $\mathrm{n}^{\circ}$ 49, p. 26 ; Pierre IRIGOIN, Les oratoires de Provence. op. cit. p. 28.

(61) "On a pu parler de "privatisation" au sens d'un repli de la religion sur la vie privée et de maintien d'un religieux invisible»». (François-André ISAMBERT, Le sens du sacré. Fête et religion populaire. Paris, Minuit, 1982, p. 7). Cette évolution est aussi mise en évidence par la privatisation des dédicaces, par l'appauvrissement du patrimoine de noms de saints dans lequel puisent les fondateurs au moment de choisir un titulaire et par le passage de demandes d'intercession intéressant la protection de toute la communauté villageoise à des demandes privées, thèmes que nous ne pouvons développer ici. 


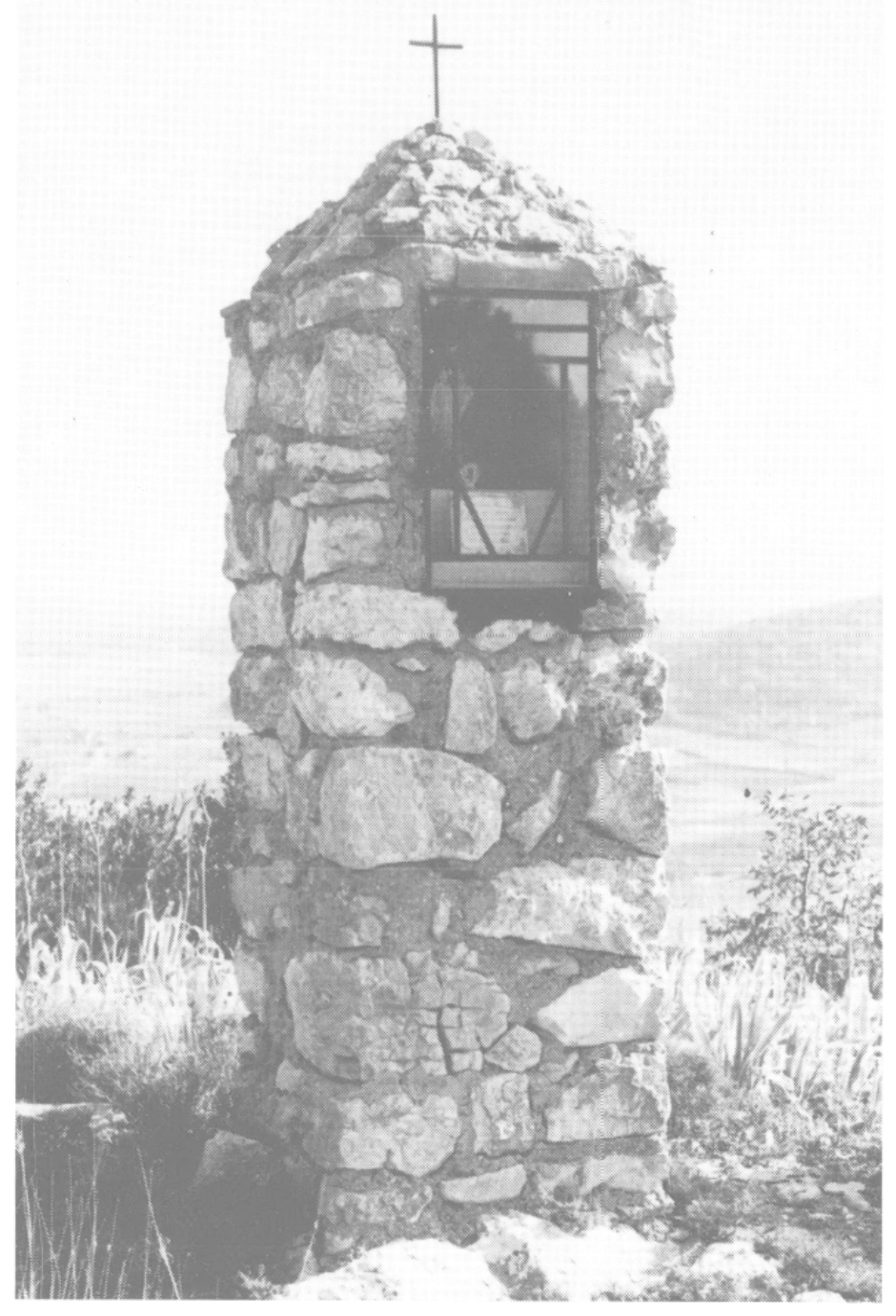

5. Notre-Dame, Saint-Julien-le-Montagnier. Oratoire ancien (1952). Localisation: saltus, bord de route. Orientation : $260^{\circ}$. Pilier en pierres; niche rectangulaire avec statuette de la Vierge protégée par un portillon vitré ; toit à quatre pentes surmonté d'une croix de fer. Inscription à l'intérieur de la niche : A toi Sainte Marie avec notre merci pour ton geste clément envers notre Maman, ce modeste oratoire en sa chère mémoire". Dimensions : $240 h \times 78 l \times 74 \mathrm{p}$. Niche $: 60 \mathrm{~h} \times 40 l \times$ $60 p$.

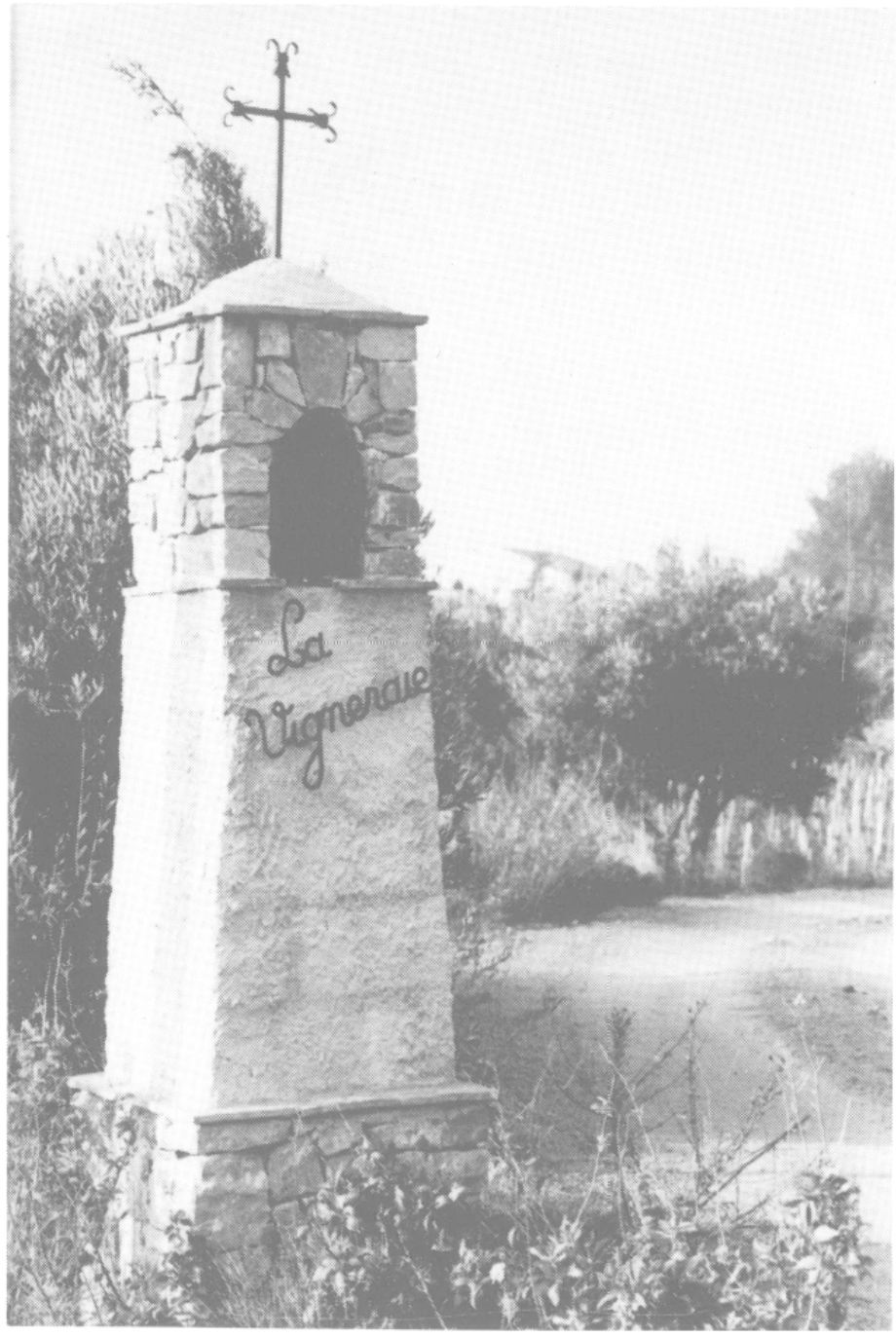

6. Saint-Etienne, Le Beausset. Oratoire récent (1979). Localisation : ager, bord de route. Orientation : $45^{\circ}$. Socle en pierres; pilier trapézoïdal crépi ; niche en pierres avec statuette et grille. Toit en coupole, croix en fer. Le pilier sert de support au nom d une villa. Dimensions : $230 h \times 60 l \times 60 p(a ̀$ la base $)$ et $50 l \times 50 p(a u$ sommet). Niche $: 34 \mathrm{~h} \times 2.5 \mathrm{I} \times .31 \mathrm{p}$. 

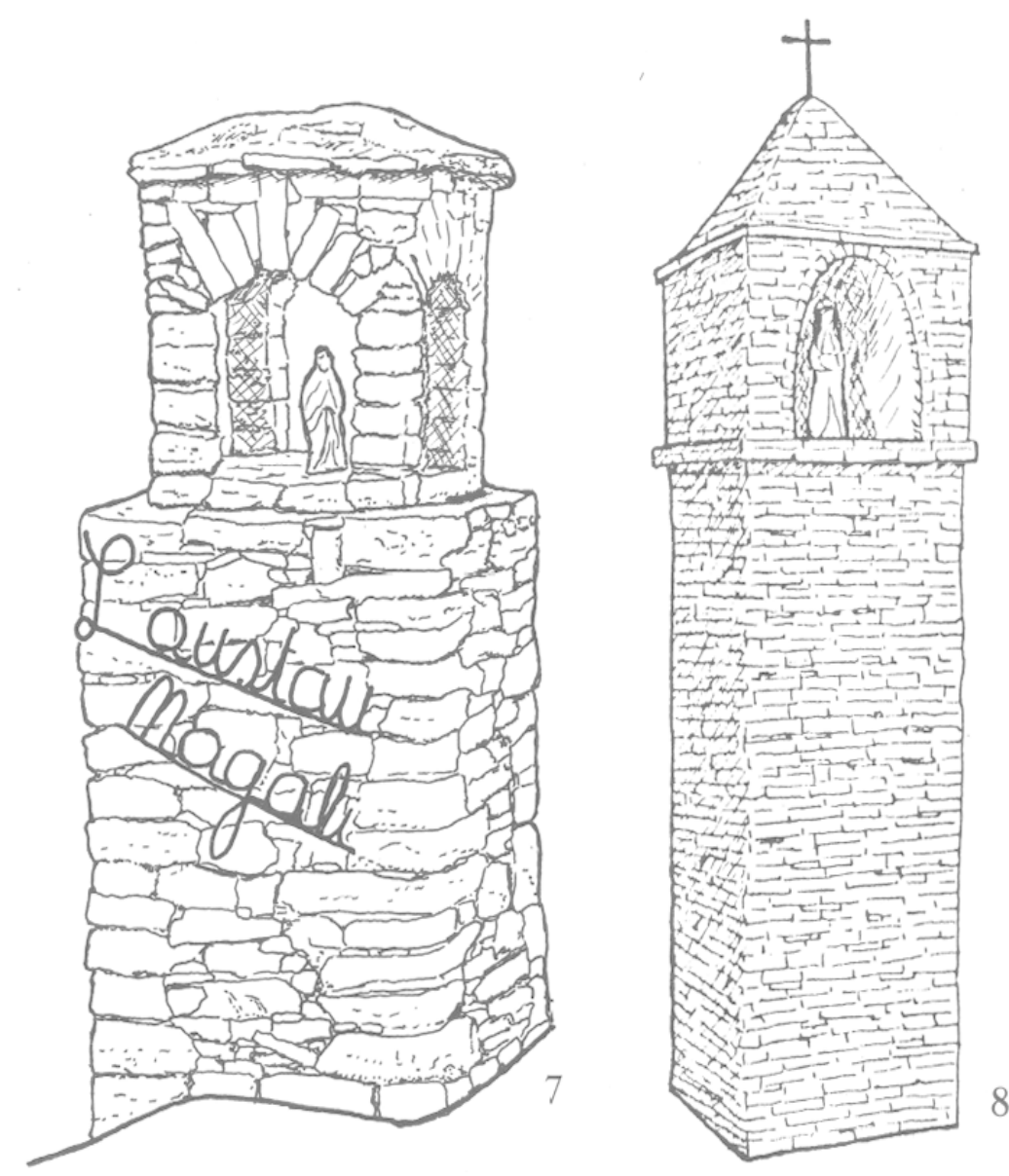

7. Saint-Clair, Le Castellet. Oratoire récent (1971). Localisation: ager, privatisé (entrée d'une propriété). Monument fonctionnalisé : le fût sert de support au nom d'une villa et fait fonction de pilier d'entrée. Orientation : $90^{\circ}$. En pierres ; niche lanterne à quatre ouvertures cintrées abritant le patron du village du Castellet. Toit en pierres plates. Dimensions: $165 h \times$ $69 l \times 69 p$. Niche $: 34 h \times 28 l \times 50 p$.

8. Sainte-Elisabeth de Hongrie, Le Beausset. Oratoire récent (1980). Localisation : urbain, privatisé (dans le jardin d'une maison du village). Orientation : $90^{\circ} . \mathrm{Pi}^{-}$ lier en petites pierres calcaires finement taillées; niche cintrée sur corniche abritant la statuette; toit à quatre pentes sur corniche surmonté d'une croix en fer. Ins. cription en dos du pilier: "1980. B. Imbert". Dimensions : $250 h \times 58 l \times 58 p$. Niche $: 40 h \times 33 l \times 38 p$.

9. Saint-Jean-Baptiste, La Cadière. Oratoire récent (1964). Localisation: ager, privatisé. Orientation : $100^{\circ}$. Pilier en petites pierres, élargi à la base, niche cintrée ouverte sur trois côtés; toit conique flanqué de modillons d'angle. Statuette du saint $(27 \mathrm{~h})$. Croix torsadée au sommet. Dimensions : $259 h \times 50 l \times 85 p$ (base) et $50 l \times 48 p$ (sommet). Niche : $36 \mathrm{~h} \times 29 l \times$ $40 \mathrm{p}$.

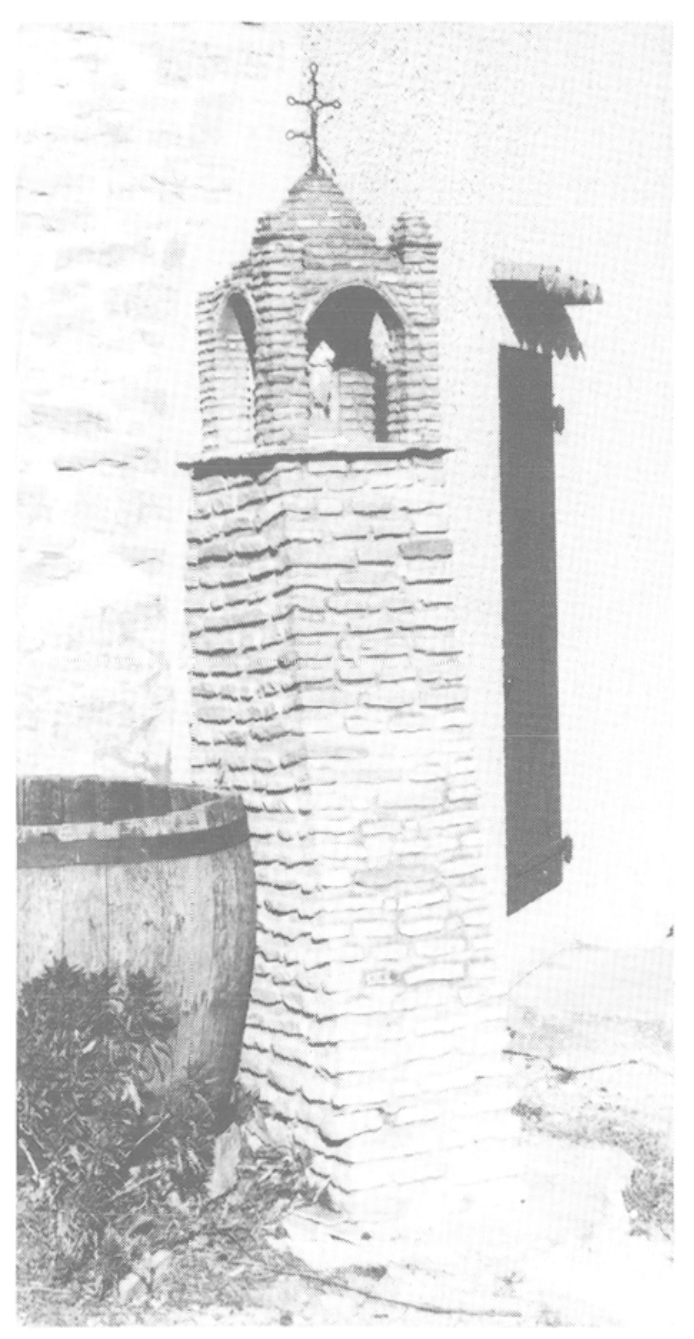


supports au nom d'une villa (Pholo 6 et Croquis 7) - marquage et emblème d'un territoire privé - alors que plusieurs oratoires anciens (62) marquaient toponymiquement l'espace communal.

De fait, les oratoires récents et privatisés sont généralement associés aux résidences principales ou secondaires, bâties en grand nombre depuis le milieu des années 1950. Localisés à l'entrée de la propriété ou dans un jardin d'agrément, ces édicules semblent vouloir donner à ce type d'habitat la touche régionale qui lui fait bien souvent défaut (63). Sans doute trouve-t-on une plus grande densité d'oratoires récents dans le canton du Beausset que dans celui de Rians parce que le premier, proche du littoral, est davantage touché par l'urbanisation (64). Si l'on admet cette perspective, on peut alors voir dans la construction des nouveaux monuments un effet de l'enrichissement, de l'apport d'argent (65) :

"Posséder un oratoire privé à l'entrée ou dans le parc de sa maison est un signe, parmi d'autres, d'aisance et de prestige" (66).

Ceci suggère une dérive profane des monuments récents que confirment effectivement leur fonctionnalisation et leur esthétisation. Celle-ci est manifeste au vu des nombreux nouveaux oratoires en pierres apparentes parfaitement agencées. ornés parfois de grilles en fer forgé devant la niche, contrastant fortement avec les anciens monuments, souvent en moellons liés au mortier de chaux et grossièrement jointoyés. L’appel fait aujourd'hui à des matériaux extéricurs aux cantons (67) (granit d'Allemagne, porphyre bleu du Dramont, pierre de Rognes), suggère un mode de construction somptuaire qui tranche avec la définition du monument classique, "d'une simplicité quasiment rudimentaire" (68).

La fonctionnalisation se vérifie lorsque le fondateur ajoute un objet fonctionnel sur l'édicule (boîte aux lettres, lanterne, portail, robinet, nom de la maison), com-

(62) Dans les deux cantons réunis, nous avons dénombré 19 édicules dont la dédicace correspond directement à un hagiotoponyme désignant un quartier. un chemin ou une route. Ce phénomène concerne 18 oratoires anciens et un seul monument récent. Jadis, oratoires et hagiotoponymes réunis constituaient des repères pour la communauté. Se déplacer dans une commune, c'était "passer simplement de la mouvance de Saint-Pierre à celle de Saint-Julien ou de celle de Saint-Joseph à celle de Sainte-Anne". (Lucienne ROUBIN, "Ordonnance toponymique en montagne provençale», L'homme hier et aujourd'hui. Recueil d'études en hommage à Leroi-Gourhan. Paris, Cujas, 1973, p. 472). Aujourd'hui, on assiste à une disparition de la "fonction spatiale des noms de saints" évoquée par Alban BENSA, op. cit.. p. 228.

(63) Dans le département comme dans les deux cantons, la période 1965.1974 a connu le plus grand nombre d'érections d'oratoires récents. Elle est aussi l'époque pendant laquelle on construisit le plus de résidences secondaires : progression de $84 \%$ de ces habitations de 1962 à 1975 dans le canton de Rians. et de $89 \%$ dans celui du Beausset.

(64) En 1975, il y avait 2823 résidences secondaires dans le canton du Beausset et 1319 dans celui de Rians.

(65) Cette question gagnerait à être approfondic. Ne peut-on considérer l'oratoire comme la chapelle privée de la classe moyenne ? Parmi les nombreuses fondations de monuments pieux qui suivirent les malheurs de la Grande Guerre. les plus fréquentes furent celles d'oratoires parce qu'elles étaient les moins coûteuses. (G. ARNAUD D’AGNEL, Léopold DOR, Les oratoires de Provence. Cannes, éd. Delonnoy, 1938, p. 51). Inversement, dans le Chablais, les familles nobles n'ont jamais érigé d'oratoires : "Il est vrai que leur fortune leur a permis de participer à la construction de chapelles et même d'églises". (G. DENAIS, R. MOLLIET, "Les oratoires du Chablais", Mémoires et Documents publiés par l'Académie Chablaisienne, t. LVIII, 1968, p. 75).

(66) Christian BROMBERGER. Jacques LACROIX, Henri RAULIN, L architecture rurale française. Corpus des genres, des types et des variantes, Paris, Berger-Levrault, 1980, p. 121.

(67) Les anciens monuments étaient bâtis essentiellement avec des matériaux (pierres, plâtre rouge) extraits de la région.

(68) Henri ALGOUD, "Les oratoires en Provence», L'art populaire en France, 1933, p. 170. 
me si les monuments construits de nos jours ne se suffisaient plus en tant que tels et avaient besoin d'être aussi des monuments pratiques, fonctionnels. Cette fonctionnalisation (69) des oratoires affecte un seul des 112 monuments anciens et 15 des 65 édicules récents, soit $23 \%$ d'entre eux. De plus, trois fonctionnalisations récentes d'oratoires anciens montrent qu'il s'agit bien d'un phénomène nouveau.

Ces différents éléments mettent en lumière une laïcisation attestée également par la disparition plus ou moins prononcée des attributs religieux (croix, bénitiers, agenouilloirs) (70) sur les monuments récents. Cette évolution est d'ailleurs transparente lorsque les fondateurs de ces oratoires affirment les avoir érigés parce que "ça fait provençal» ou parce que "ça va avec le paysage».

Rćsumons-nous. L'oratoire ancien, public ct aisćment accessible, situé au bord d'un champ ou d'un chemin conduisant à une chapelle rurale, était lié à la vie paysanne (71) et aux activités pérégrines. L'oratoire récent, érigé à l'entrée ou à l'intérieur d'une propriété privée, semble un effet du développement de la fonction résidentielle. Certes, les deux monuments révèlent, à des degrés différents, la permanence de la dulie des saints dans le département du Var. Mais il n'empêche que l'on est bien passé du monument qui "rassure et console» (72), de l'édicule conjuratoire des angoisses eschatologiques et des frayeurs terrestres les plus diverses (menaces sur les récoltes, maladie, etc.), à un monument privatisé, esthétisé et fonctionnalisé. Cet oratoire laïcisé, folklorisé pourrait-on dire, est l'image, semble-t-il, d'une société en voie de déruralisation, en train de rompre avec tout un héritage de sacralité.

Il est banal de soutenir l'impossibilité d'étudier une tradition dite populaire en l'isolant des bouleversements socio-économiques qui affectent la société globale. Notre recherche sur les oratoires en apporte, pensons-nous, une nouvelle démonstration. Le choix, comme critère de bipartition du corpus, du milieu des années 1950. époque où s'est accentué le processus de déruralisation, a en effet été validé a posteriori. Il a permis de mettre en évidence un nouvel usage d'un monument traditionnel, sous l'influence de nouvelles conditions socio-économiques (73). Cela confirme, comme l'a bien noté J.-C. Schmitt, qu'une tradition est toujours vécue et jamais survécue (74).

\author{
Joël CANDAU, \\ Saint-Laurent-du-Var
}

(69) Ce terme ne veut pas dire que les oratoires ont pour fonction, par exemple, de servir de boîte aux lettres car, si cela était, on ne voit pas pourquoi les fondateurs ne bâtiraient pas de simples piliers en pierre. Nous voulons signifier simplement le rajout d'un objet fonctionnel, pratique, sur l'édicule.

(70) La statuette du titulaire, qui n'est pas en régression, occupe une place à part : appelé "santon" par certains fondateurs de monuments, cet objet peut difficilement être compté comme un indicateur du caractère religieux du monument, car il appartient à l'image folklorisante de la Provence véhiculée par la crèche. En ce sens, sa fréquence plus grande au sein des oratoires récents exprime davantage la recherche d'un objet qui «fait provençal» que l'ardeur de la dévotion au saint.

(71) Alors que $84 \%$ des fondateurs connus des monuments anciens étaient des agriculteurs, $67 \%$ des fondateurs d'édicules récents sont des non-agriculteurs. De plus, ces derniers sont à l'origine de la quasi-totalité des oratoires esthétisés et fonctionnalisés.

(72) Pierre IRIGOIN, Les oratoires de Provence, op. cit., p. 7.

(73) Il n'est pas sans intérêt de noter que les oratoires ne sont pas les seuls monuments à avoir connu une mutation dans le milieu des années 1950. Dans son étude des symboles ornant les tombes, JeanOlivier MAJASTRE fait la même observation et note, à cette époque-là, une rupture complète avec le style funéraire traditionnel ( "L'espace des morts et le monde des vivants", Le monde alpin et rhodanien, $n^{\circ} 1-4 / 1977$, p. 247-269).

(74) Jean-Claude SCHMITT, "Religion populaire et culture folklorique», Annales ESC, sept.-oct. $976, n^{\circ} 5$, p. 946. 
ALGOUD (Henri), "Les oratoires en Provence», L art populaire en France, Cinquième année, 1933, pp. 167-175.

ARNAUD D'AGNEL (G.), DOR (Léopold), Les oratoires de Provence, Cannes, éd. Delonnoy, 1938, 122 p.

BRUN (Louis), Oratoires de nos terroirs. Cantons de Bourg-Saint-Andéol (Ardèche), Pont-Saint-Esprit, Bagnols-sur-Cèze (Gard), Nîmes, Louis Brun, 1976, $71 \mathrm{p}$.

CELIS (Gabriel), Les édicules pieux en Belgique, Gand. Les Amis de la Sainte Vierge, s.d., 24 p.

CHIDE (Alphonse), "Les oratoires de Provence", La revue du Touring Club de France, $\mathrm{n}^{\circ} 458$, janvier 1933, pp. 27-31.

DENAIS (Gilbert), MOLLIET (Robert), «Les oratoires du Chablais", Mémoires et Documents publiés par l'Académie Chablaisienne, t. LVIII, 1968.

DUFOURNET (Paul), "Les oratoires de Savoie», $L$ 'art populaire en France, Sixième année, 1934-1935. pp. 139-182.

DUFOURNET (Paul), "Les oratoires de Savoie", Revue Savoisienne, $1953,1^{\text {er }}$ et $2^{\mathrm{e}}$ trim., pp. 44-66.

FILIPPI (André), MARTEL (André), Les oratoires de la Provence varoise, éd. des Cariatides, 1935, 14 p.

GAVOT (Jean), Le Beausset. Par les champs et les collines. A travers les places et les rues. Dans la mystique et la tradition des oratoires. Le Beausset, éd. Atelier du Beausset, 1982, ill., 107 p.

GAVOT (Jean), Les oratoires bas-alpins, Aix-en-Provence, Amis des Oratoires, s.d., 54 p.

HENSELING (Louis), "Les oratoires du Var», Bull. de la Société des Amis du Vieux Toulon, $\mathrm{n}^{\circ} 49$, janv.fév.-mars 1936, pp. 23-35.

'HENSELING (Louis), Les oratoires du Var. Toulon. Impr. F. Cabasson, s.d., 52 p.

HENSELING (Louis), "Les oratoires du Var", in Zigzags dans le Var, 1934, pp. 117-169.

HENSELING (Louis), "San Sumian (cippe gallo-romain de la banlieue de Brignoles)", Bull. de la Société des Amis du Vieux Toulon, 1932, n 36, p. 206.

IRIGOIN (Pierre), "Les oratoires de Provence classés par Communes", Mémoires de l'Institut historique

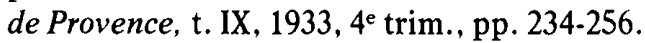

IRIGOIN (Pierre), "Montjoies et oratoires", Bulletin Monumental, 1935, 2, pp. 147-170.

IRIGOIN (Pierre), "Les oratoires de la Sainte-Baume", in Almanach de Provence et du Comté de Nice, 1937, St Félicien (Ardèche), éd. du Pigeonnier, 1936, pp. 71-75 (159 p.).

IRIGOIN (Pierre), "Les oratoires des Alpes», Folklore paysan, $\mathrm{n}^{\circ} 3$, mai-juin-juill. 1939, pp. 79-81.

IRIGOIN (Pierre), Les oratoires de la Sainte-Baume, Aix-en-Provence, Amis des Oratoires, 1940, 53 p.

IRIGOIN (Pierre), Les oratoires des Bouches-du-Rhône, Aix-en-Provence, Amis des Oratoires, 1947, 70 p.

IRIGOIN (Pierre), Les oratoires de France depuis les origines, Aix-en-Provence, Amis des Oratoires. 1977, III, dessins de Pierre LHUILLIER, préface de Jean GAVOT, 325 p.

JALLON (R.), Les oratoires des Alpes-Maritimes, s.l., sept. 1942.

JANVIER (Louis), Inventaire des oratoires $d u$ Var, Aixen-Provence, Amis des Oratoires, 1982, Index, ill., $121 \mathrm{p}$.

JOLIN (René), Inventaire des chapelles de Pierre Bleue du Hainaut, Metz, Est Imprimerie, 1972, ill., 59 p.

LAUGIER (E.F.), GAVOT (Jean), «Oratoires ; Niches et Chapelles d'arbres", Arts et Traditions populaires, janv.-juin 1969, n 1-2, tiré à part, 14 p.

MAUNIER (J.), VALENTIN (R.), JALLON (R.), ANGELINI (L.), Les oratoires des Alpes-Maritimes, Aixen-Provence, Amis des Oratoires, sept. 1942, 22 p.

MILON (Yves), Fontaines en Bretagne, Paris, Plon, 1964 , ill., cartes, $106 \mathrm{p}$.

PAOLI (J.L.), Les oratoires de Haute-Provence, multigraphié, 1982, XIII - 56 p.

PAUPLIN (René), Les oratoires $d u$ Vaucluse, Aix-en. Provence, Amis des Oratoires, 1970, Index, 127 p.

PIDOUX de la MADUERE (André), "Les Oratoires de la Franche-Comté», Le Pays Comtois, $\mathrm{n}^{\circ} 101$, fév. 1937, pp. 73-76.

PIGUILLEM (V.), "Les oratoires des Pyrénées orientales", Archistra, n 13, 1974, pp. 5-21.

PORCHERON (Louis), "Les oratoires de Provence", Ciels et terres de Provence, Marseille, éd. "Quo vadis", 1938, $432 \mathrm{p}$. 
SCRIVE-LOYER (J.), Le Montjoie de Notre-Dame de Bonne Espérance à Vellereille de Bayeux, près de Buiche (Belgique). Avesnes-sur-Helpe, éd. de l'observateur, 1935, Bibliogr., 24 p.

VALLENTIN DU CHEYLARD (Raymond), "Les oratoires de la Drôme», Bull. de l'Association "Les Amis du Buis et des Baronnies», n 9, 1971, pp. 6-17.
VAN GENNEP (Arnold), «Patronages, chapelles et oratoires de la Haute-Maurienne. Étude statistique et critique", Tiré à part de la Revue d'Histoire de l'Eglise de France, t. XXV, 1939, pp. 145-182.

VIAL-MAZEL (Geneviève), Les oratoires des Alpes sur la mer, Aix-en-Provence, Amis des Oratoires, 1962, Cartes, ill., 133 p.

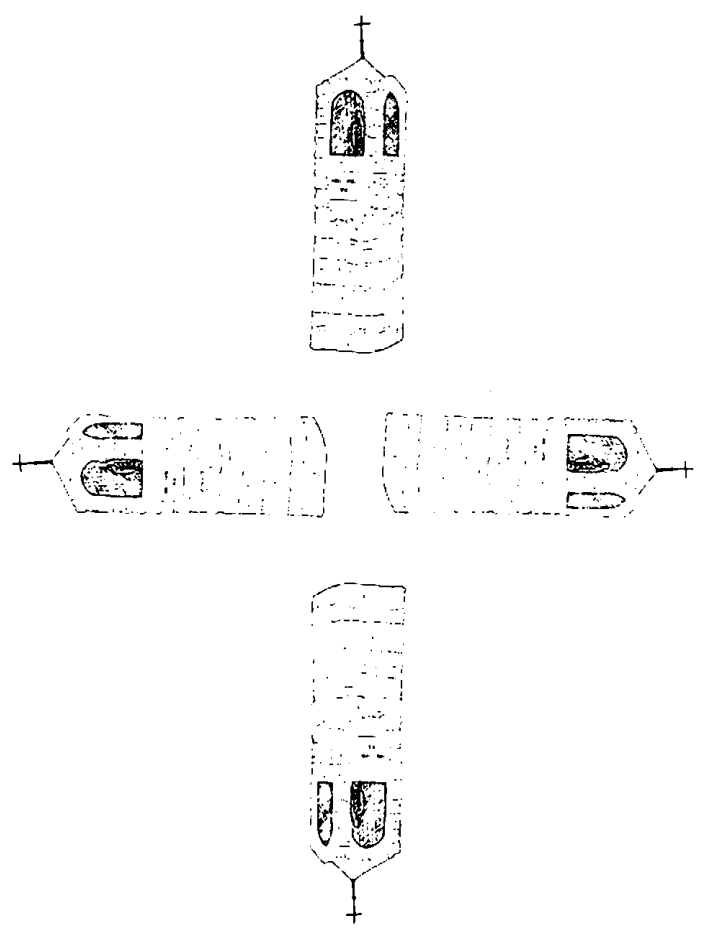

